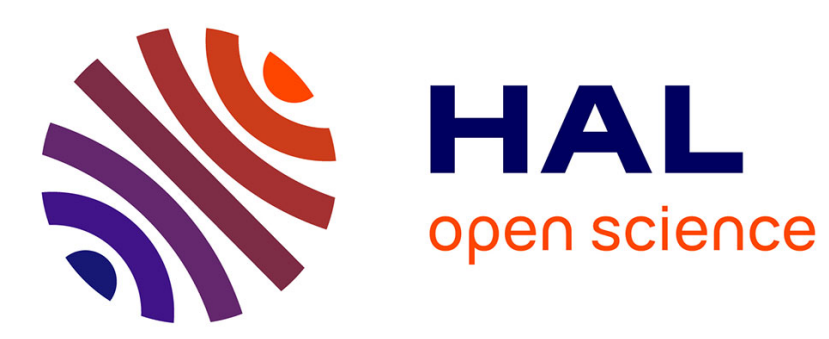

\title{
Local distribution of the parts of unequal partitions in arithmetic progressions II
}

Cécile Dartyge, Mihály Szalay

\section{To cite this version:}

Cécile Dartyge, Mihály Szalay. Local distribution of the parts of unequal partitions in arithmetic progressions II. Publicationes Mathematicae Debrecen, 2012, 81 (3-4), pp.453-486. 10.5486/PMD.2012.5332 . hal-01280666

\section{HAL Id: hal-01280666 https://hal.science/hal-01280666}

Submitted on 29 Feb 2016

HAL is a multi-disciplinary open access archive for the deposit and dissemination of scientific research documents, whether they are published or not. The documents may come from teaching and research institutions in France or abroad, or from public or private research centers.
L'archive ouverte pluridisciplinaire HAL, est destinée au dépôt et à la diffusion de documents scientifiques de niveau recherche, publiés ou non, émanant des établissements d'enseignement et de recherche français ou étrangers, des laboratoires publics ou privés. 


\title{
Local distribution of the parts of unequal partitions in arithmetic progressions II
}

\author{
Cécile Dartyge (Nancy) and Mihály Szalay (Budapest) *
}

\section{Introduction}

This paper contains the main parts of the proofs of the results announced in [6]. We recall below some notations and the main result of this paper but we recommend to the reader to study first [6]. Let $d \in \mathbb{N}^{*}, \mathcal{D}$ a non-empty subset of $\{1, \ldots, d\}$ and $\mathcal{D}^{c}=\{1, \ldots, d\} \backslash \mathcal{D}$ its complement. Let $\mathcal{R}_{\mathcal{D}}=\left\{N_{r}: r \in \mathcal{D}\right\}$ be a multiset of $|\mathcal{D}|$ non-negative integers. The main goal of our work is to obtain an asymptotic formula for $\Pi_{d}^{*}\left(n, \mathcal{R}_{\mathcal{D}}\right)$, the number of unequal partitions of $n$ with exactly $N_{r}$ parts congruent to $r$ modulo $d$ for all $r \in \mathcal{D}$. We adopt the convention $\Pi_{d}^{*}\left(0, \mathcal{R}_{\mathcal{D}}\right)=1$ if $\mathcal{R}_{\mathcal{D}}=\{0, \ldots, 0\}$ and 0 otherwise.

Recall that if $n \geqslant 1$ and $\Pi_{d}^{*}\left(n, \mathcal{R}_{\mathcal{D}}\right) \geqslant 1$ then $n$ satisfies

$$
n \equiv R_{\mathcal{D}}(\bmod \delta)
$$

where $R_{\mathcal{D}}=\sum_{r \in \mathcal{D}} r N_{r}$ and $\delta$ is the $g . c$. $d$. of the elements of $\mathcal{D}^{c} \cup\{d\}$. In the introduction of [6], we observed that the $N_{r}, r \in \mathcal{D}$ may be expected to be close to $k_{0}$ with

$$
k_{0}:=\frac{2 \sqrt{3} \log 2}{\pi} \frac{\sqrt{n}}{d} .
$$

More precisely we suppose that for all $r \in \mathcal{D}$ we have

$$
\left|N_{r}-k_{0}\right| \leqslant \frac{n^{\frac{1}{4}} \sqrt{\log n}}{d^{1 / 3}|\mathcal{D}|^{2 / 3} w(n)},
$$

where $w(n)$ is a non-decreasing function such that $w(n) \rightarrow \infty$ if $n \rightarrow \infty$. Let us recall the main result of $[6]$

Theorem 1.1. Let $\varepsilon>0$. The following two propositions hold.

(i) Let $d \leqslant n^{1 / 4-\varepsilon}, \mathcal{D}=\{1, \ldots, d\}$ and $n \equiv R_{\mathcal{D}}(\bmod d)$. Let $\mathcal{R}=\mathcal{R}_{\mathcal{D}}=\left\{N_{1}, \ldots, N_{d}\right\}$ be a multiset of integers satisfying $(1 \cdot 3)$. Then we have

$$
\begin{aligned}
& \Pi_{d}^{*}\left(n, \mathcal{R}_{D}\right)=(1+o(1)) q(n) \frac{d}{\sqrt{1-\frac{12(\log 2)^{2}}{\pi^{2}}}}\left(\frac{d}{2 \sqrt{3 n}}\right)^{d / 2} \\
& \quad \times \exp \left\{-\frac{2 \sqrt{3} \log ^{2} 2}{\pi\left(1-\frac{12(\log 2)^{2}}{\pi^{2}}\right) \sqrt{n}}\left(\sum_{r=1}^{d}\left(N_{r}-k_{0}\right)\right)^{2}-\frac{\pi d}{2 \sqrt{3 n}} \sum_{r=1}^{d}\left(N_{r}-k_{0}\right)^{2}\right\} .
\end{aligned}
$$

(ii) We suppose now that $d \leqslant n^{1 / 6-\varepsilon}$ and $\mathcal{D} \subset\{1, \ldots, d\}$. Then under $(1 \cdot 1)$ and $(1 \cdot 3)$ we have

$$
\begin{aligned}
\Pi_{d}^{*}\left(n, \mathcal{R}_{D}\right) & =q(n) \frac{\delta(1+o(1))}{\sqrt{1-\frac{12|\mathcal{D}|(\log 2)^{2}}{d \pi^{2}}}}\left(\frac{d}{2 \sqrt{3 n}}\right)^{|\mathcal{D}| / 2} \\
& \times \exp \left(-\frac{2 \sqrt{3}(\log 2)^{2}}{\pi\left(1-\frac{12|\mathcal{D}|(\log 2)^{2}}{d \pi^{2}}\right) \sqrt{n}}\left(\sum_{r \in \mathcal{D}}\left(N_{r}-k_{0}\right)\right)^{2}-\frac{\pi d}{2 \sqrt{3 n}} \sum_{r \in \mathcal{D}}\left(N_{r}-k_{0}\right)^{2}\right) .
\end{aligned}
$$

Mathematics Subject Classification (2010): 11P82; 05A17, 11P83.

* Research partially supported by the Hungarian National Foundation for Scientific Research, Grant K 67676 and by French-Hungarian EGIDE-OMKFHÁ exchange program Balaton FR$33 / 2009$. 
First we complete the proof of Theorem 1.1 in the case $\mathcal{D}=\{1, \ldots, d\}$, after we will handle the complementary case when $\mathcal{D}^{c} \neq \varnothing$. The last sections are devoted to the proofs of the different corollaries of [6].

\section{The term $S_{2}$}

We begin to assume that

$$
d \leqslant n^{\frac{1}{2}-\varepsilon}
$$

with some fixed positive $\varepsilon$ and

$$
\left|k-k_{0}\right|=o\left(\frac{\sqrt{n}}{d}\right)
$$

Let

$$
x_{0}:=\frac{\pi}{2 \sqrt{3 n}}, \quad t:=d x_{0} .
$$

Then

$$
k_{0} t=k_{0} d x_{0}=\log 2 .
$$

We also suppose that

$$
\left|N_{r}-k_{0}\right|=o\left(\frac{\sqrt{n}}{d}\right) \quad(r=1, \ldots, d) .
$$

In Section 4 of [6] we proved that as $n \rightarrow \infty$ then we have

$$
\prod_{r=1}^{d} g_{N_{r}}\left(d x_{0}\right)=\exp \left(\frac{\pi^{2}}{12 x_{0}}+\frac{(\log 2)^{2}}{2 x_{0}}+o(\sqrt{n})\right)
$$

According to the notations of [6] Sections 3 and 4, we have

$$
\left|S_{2}\right| \leqslant \frac{d}{2 \pi} \int_{3 \pi x_{0} \leqslant|y| \leqslant \pi / d}\left\{\prod_{r=1}^{d}\left|g_{N_{r}}\left(d\left(x_{0}+i y\right)\right)\right|\right\} \exp \left((n-R-Q) x_{0}\right) \mathrm{d} y .
$$

The main part of this section is the following lemma

Lemma 2.1. Under the notations and hypotheses (1.2), (2.1), (2.2), (2.3), (2.4), and $3 \pi x_{0} \leqslant|y| \leqslant \pi / d$, we have

$$
\left|g_{k}\left(d\left(x_{0}+i y\right)\right)\right| \leqslant g_{k}\left(d x_{0}\right) \exp \left(\frac{-1+o(1)}{4 d x_{0}}\right) .
$$

Proof. This time we start out from the first expression of $g_{k}$ and develop the logarithms:

$$
\begin{aligned}
g_{k}(w) & =\exp \left(\sum_{\nu=1}^{k} \log \frac{1}{1-\exp (-\nu w)}\right)=\exp \left(\sum_{\nu=1}^{k} \sum_{m=1}^{\infty} \frac{1}{m} \exp (-\nu m w)\right) \\
& =\exp \left(\sum_{m=1}^{\infty} \frac{1}{m} \sum_{\nu=1}^{k} \exp (-\nu m w)\right)=\exp \left(\sum_{\nu=1}^{k} \exp (-\nu w)+\sum_{m=2}^{\infty} \frac{1}{m} \sum_{\nu=1}^{k} \exp (-\nu m w)\right) .
\end{aligned}
$$


We take the moduli

$$
\begin{aligned}
\left|g_{k}(w)\right| & \leqslant \exp \left(\left|\sum_{\nu=1}^{k} \exp (-\nu w)\right|+\sum_{m=2}^{\infty} \frac{1}{m} \sum_{\nu=1}^{k} \exp (-\nu m t)\right) \\
& =g_{k}(t) \exp \left(\left|\sum_{\nu=1}^{k} \exp (-\nu w)\right|-\sum_{\nu=1}^{k} \exp (-\nu t)\right) \\
& =g_{k}(t) \exp \left(\frac{|1-\exp (-k w)|}{|\exp (w)-1|}-\frac{1-\exp (-k t)}{\exp (t)-1}\right) \\
& \leqslant g_{k}(t) \exp \left(\frac{1+\exp (-k t)}{|\exp (w)-1|}-\frac{1-\exp (-k t)}{\exp (t)-1}\right) .
\end{aligned}
$$

When $|\Im m w| \leqslant \pi$,

$$
|\exp (w)-1|^{2}=(\exp (t)-1)^{2}+4 \mathrm{e}^{t}(\sin (b / 2))^{2} \geqslant 4(\sin (b / 2))^{2} \geqslant \frac{4|b|^{2}}{\pi^{2}}
$$

thus

$$
\left|g_{k}(w)\right| \leqslant g_{k}(t) \exp \left(\frac{1+\exp (-k t)}{\frac{2}{\pi}|\Im m w|}-\frac{1-\exp (-k t)}{\exp (t)-1}\right)
$$

if $|\Im m w| \leqslant \pi$. Therefore, $3 \pi x_{0} \leqslant|y| \leqslant \pi / d$ implies that

$$
\begin{aligned}
\left|g_{k}\left(d\left(x_{0}+i y\right)\right)\right| & \leqslant g_{k}\left(d x_{0}\right) \exp \left(\frac{1+\exp \left(-k d x_{0}\right)}{\frac{2}{\pi} d|y|}-\frac{1-\exp \left(-k d x_{0}\right)}{\exp \left(d x_{0}\right)-1}\right) \\
& \leqslant g_{k}\left(d x_{0}\right) \exp \left(\frac{1+\exp \left(-k d x_{0}\right)}{6 d x_{0}}-\frac{1-\exp \left(-k d x_{0}\right)}{\exp \left(d x_{0}\right)-1}\right)
\end{aligned}
$$

By $(1 \cdot 2),(2 \cdot 1),(2 \cdot 2),(2 \cdot 3),(2 \cdot 4)$,

$$
\begin{aligned}
\left|g_{k}\left(d\left(x_{0}+i y\right)\right)\right| & \leqslant g_{k}\left(d x_{0}\right) \exp \left(\frac{\frac{3}{2}+o(1)}{6 d x_{0}}-\frac{\frac{1}{2}+o(1)}{\exp \left(d x_{0}\right)-1}\right) \\
& =g_{k}\left(d x_{0}\right) \exp \left(\frac{\frac{3}{2}+o(1)}{6 d x_{0}}-\frac{\frac{1}{2}+o(1)}{d x_{0}}+O(1)\right) \\
& =g_{k}\left(d x_{0}\right) \exp \left(-\frac{1+o(1)}{4 d x_{0}}\right)
\end{aligned}
$$

This ends the proof of Lemma 2.1.

By $(2 \cdot 5)$ we obtain for $S_{2}$,

$$
\begin{aligned}
\left|S_{2}\right| & \leqslant\left\{\prod_{r=1}^{d} g_{N_{r}}\left(d x_{0}\right)\right\} \exp \left(-\frac{1+o(1)}{4 x_{0}}\right) \exp \left((n-R-Q) x_{0}\right) \\
& =\exp \left(\frac{\pi \sqrt{n}}{\sqrt{3}}-\frac{\sqrt{3 n}}{2 \pi}+o(\sqrt{n})\right)
\end{aligned}
$$

by (2.6) and according to the estimates of $Q$ and $R$ obtained in Sections 2 and 4 of [6]. 


\section{The term $S_{1}$}

Next, we will try to give a similar and simple estimation for $n^{-\frac{5}{8}+\varepsilon} \leqslant|y| \leqslant 3 \pi x_{0}$.

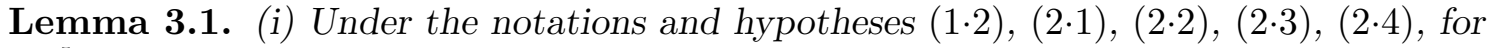
$n^{-\frac{5}{8}+\varepsilon} \leqslant|y| \leqslant 3 \pi x_{0}$ we have

$$
\left|g_{k}\left(d\left(x_{0}+i y\right)\right)\right| \leqslant g_{k}\left(d x_{0}\right) \exp \left(-\frac{\sqrt{3} n^{1 / 4+2 \varepsilon}}{27 \pi^{5} d}\right) .
$$

(ii) Under the notations and hypotheses $(1 \cdot 2),(2 \cdot 1),(2 \cdot 2),(2 \cdot 3),(2 \cdot 4)$, for $n^{-\frac{3}{4}+\frac{\varepsilon}{3}} \leqslant|y| \leqslant$ $n^{-\frac{5}{8}+\varepsilon}$ we have

$$
\left|g_{k}\left(d\left(x_{0}+i y\right)\right)\right| \leqslant g_{k}\left(d x_{0}\right) \exp \left(-\frac{\sqrt{3} n^{2 \varepsilon / 3}}{27 \pi^{5} d}\right) .
$$

Proof of (i). We suppose that $n^{-\frac{5}{8}+\varepsilon} \leqslant|y| \leqslant 3 \pi x_{0}$. By $(2 \cdot 7)$ we have

$$
g_{k}(w)=\exp \left(\sum_{\nu=1}^{k} \exp (-\nu w)+\sum_{m=2}^{\infty} \frac{1}{m} \sum_{\nu=1}^{k} \exp (-\nu m w)\right) .
$$

We study again $\left|g_{k}(w)\right|$ :

$$
\begin{aligned}
\left|g_{k}(w)\right| & \leqslant \exp \left(\Re e\left(\sum_{\nu=1}^{k} \exp (-\nu w)\right)+\sum_{m=2}^{\infty} \frac{1}{m} \exp (-\nu m t)\right) \\
& =g_{k}(t) \exp \left(\sum_{\nu=1}^{k} \Re e(\exp (-\nu w))-\sum_{\nu=1}^{k} \exp (-\nu t)\right) \\
& =g_{k}(t) \exp \left(\sum_{\nu=1}^{k} \exp (-\nu t)(\Re e \exp (-\nu i b)-1)\right) \\
& =g_{k}(t) \exp \left(\sum_{\nu=1}^{k} \exp (-\nu t)(\cos (\nu b)-1)\right) \\
& =g_{k}(t) \exp \left(-2 \sum_{\nu=1}^{k} \exp (-\nu t) \sin ^{2}\left(\frac{\nu|b|}{2}\right)\right) .
\end{aligned}
$$

Let $K_{0}:=\left\lfloor\frac{k_{0}}{3 \log 2}\right\rfloor$. If $k=k_{0}+o\left(\frac{\sqrt{n}}{d}\right)$ then $k>K_{0}$ for $n$ large enough :

$$
\begin{aligned}
\left|g_{k}(w)\right| & \leqslant g_{k}(t) \exp \left(-2 \sum_{\nu=1}^{K_{0}} \exp (-\nu t) \sin ^{2}\left(\frac{\nu|b|}{2}\right)\right) \\
& \leqslant g_{k}(t) \exp \left(-2 \sum_{\nu=1}^{K_{0}} \exp \left(-k_{0} t\right) \sin ^{2}\left(\frac{\nu|b|}{2}\right)\right)=g_{k}(t) \exp \left(-\sum_{\nu=1}^{K_{0}} \sin ^{2}\left(\frac{\nu|b|}{2}\right)\right) .
\end{aligned}
$$

Writing $w=d\left(x_{0}+i y\right)$ and using the inequality $|\sin t| \geqslant \frac{2|t|}{\pi}$ for $|t| \leqslant \pi / 2$, we obtain

$$
\left|g_{k}\left(d\left(x_{0}+i y\right)\right)\right| \leqslant g_{k}\left(d x_{0}\right) \exp \left(-\sum_{\nu=1}^{K_{0}}\left(\frac{\nu d|y|}{\pi}\right)^{2}\right)
$$

since

$$
\frac{\nu d|y|}{2} \leqslant \frac{K_{0} d 3 \pi x_{0}}{2} \leqslant \frac{k_{0} d \pi x_{0}}{2 \log 2}=\frac{\pi}{2}
$$


Since $\sum_{\nu=1}^{K_{0}} \nu^{2} \geqslant \frac{K_{0}^{3}}{3}$, we have

$$
\left|g_{k}\left(d\left(x_{0}+i y\right)\right)\right| \leqslant g_{k}\left(d x_{0}\right) \exp \left(-\left(\frac{d|y|}{\pi}\right)^{2} \frac{K_{0}^{3}}{3}\right) \leqslant g_{k}\left(d x_{0}\right) \exp \left(-\frac{\sqrt{3} n^{1 / 4+2 \varepsilon}}{\pi^{5} 3^{3} d}\right)
$$

(ii) can be obtained similarly.

By $(2 \cdot 5)$,

$$
\begin{aligned}
& \left|\frac{d}{2 \pi} \int_{n^{-\frac{5}{8}+\varepsilon} \leqslant|y| \leqslant 3 \pi x_{0}}\left\{\prod_{r=1}^{d} g_{N_{r}}\left(d\left(x_{0}+i y\right)\right)\right\} \exp \left((n-R-Q)\left(x_{0}+i y\right)\right) \mathrm{d} y\right| \\
& \leqslant \frac{d}{2 \pi} \int_{n^{-\frac{5}{8}+\varepsilon} \leqslant|y| \leqslant 3 \pi x_{0}}\left|\prod_{r=1}^{d} g_{N_{r}}\left(d\left(x_{0}+i y\right)\right)\right| \exp \left((n-R-Q) x_{0}\right) \mathrm{d} y \\
& \leqslant\left\{\prod_{r=1}^{d} g_{N_{r}}\left(d x_{0}\right)\right\} \exp \left(-\frac{\sqrt{3} n^{\frac{1}{4}+2 \varepsilon}}{\pi^{5} 3^{3}}\right) \exp \left((n-R-Q) x_{0}\right) .
\end{aligned}
$$

We have to stop here since the previously error term $o(\sqrt{n})$ is rough. Otherwise the above proof can be applied, e. g., for $n^{-\frac{3}{4}+\frac{\varepsilon}{3}} \leqslant|y| \leqslant n^{-\frac{5}{8}+\varepsilon}$ and results that

$$
\begin{aligned}
& \left|\frac{d}{2 \pi} \int_{n^{-\frac{3}{4}+\frac{\varepsilon}{3}} \leqslant|y| \leqslant n^{-\frac{5}{8}+\varepsilon}}\left\{\prod_{r=1}^{d} g_{N_{r}}\left(d\left(x_{0}+i y\right)\right)\right\} \exp \left((n-R-Q)\left(x_{0}+i y\right)\right) \mathrm{d} y\right| \\
& \leqslant\left\{\prod_{r=1}^{d} g_{N_{r}}\left(d x_{0}\right)\right\} \exp \left(-\frac{\sqrt{3} n^{2 \varepsilon / 3}}{\pi^{5} 3^{3}}\right) \exp \left((n-R-Q) x_{0}\right) .
\end{aligned}
$$

Finally we obtain for $S_{1}$ :

$$
\left|S_{1}\right| \ll\left\{\prod_{r=1}^{d} g_{N_{r}}\left(d x_{0}\right)\right\} \exp \left(-\frac{\sqrt{3} n^{2 \varepsilon / 3}}{\pi^{5} 3^{3}}\right) \exp \left((n-R-Q) x_{0}\right) .
$$

\section{The function $g_{k}$ in the range $|y|<y_{1}$.}

Let $|y| \leqslant y_{1}=n^{-\frac{3}{4}+\frac{\varepsilon}{3}}, w=t+i b=d x_{0}+i d y$. Now $\frac{|b|}{t}=O\left(n^{-\frac{1}{4}+\frac{\varepsilon}{3}}\right)$.

In this section we work with a general subset $\mathcal{D} \subset\{1, \ldots, d\}$.

Instead of $(2 \cdot 2)$ and $(2 \cdot 5)$, we suppose that

$$
\left|k-k_{0}\right| \leqslant \frac{n^{\frac{1}{4}} \sqrt{\log n}}{d^{1 / 3}|\mathcal{D}|^{2 / 3} w(n)} \quad \text { and } \quad\left|N_{r}-k_{0}\right| \leqslant \frac{n^{\frac{1}{4}} \sqrt{\log n}}{d^{1 / 3}|\mathcal{D}|^{2 / 3} w(n)} \quad(r \in \mathcal{D})
$$

where $w(n)$ is a non-decreasing function such that $w(n) \rightarrow \infty$ if $n \rightarrow \infty$. The aim of this paragraph is to obtain an asymptotic formula for $g_{k}\left(x_{0}+i y\right)$ for $|y| \leqslant y_{1}$. Instead of $(2 \cdot 1)$, we suppose that

$$
d \leqslant n^{\frac{1}{4}-2 \varepsilon}
$$

Thus (4.1) implies $(2 \cdot 2)$ and $(2 \cdot 5)$. We will prove the following Lemma: 
Lemma 4.1. Under (4.1) we have

$$
\begin{aligned}
g_{k}(w) & =f(w) \exp \left\{-\frac{C_{2}}{t}+\left(k-k_{0}\right) \log 2-\frac{\left(k-k_{0}\right)^{2} t}{2}+\frac{\log 2}{2}\right. \\
& \left.+i b\left(\frac{C_{2}}{t^{2}}+\frac{k_{0} \log 2}{t}-k_{0}\left(k-k_{0}\right)\right)+b^{2}\left(\frac{C_{2}}{t^{3}}+\frac{k_{0} \log 2}{t^{2}}+\frac{k_{0}^{2}}{2 t}\right)+o\left(\frac{n^{-\varepsilon}}{|\mathcal{D}|}\right)\right\} .
\end{aligned}
$$

We have again by Lemma 4.1 of [6]

$$
g_{k}(w)=f(w) \exp \left\{-\frac{1}{w} \sum_{m=1}^{\infty} \frac{1}{m^{2}} \exp (-k m w)+\frac{1}{2} \sum_{m=1}^{\infty} \frac{1}{m} \exp (-k m w)+O\left(k^{-1}\right)\right\} .
$$

By $(1 \cdot 2)$ and $(2 \cdot 2)$,

$$
\frac{1}{k}=O\left(\frac{d}{\sqrt{n}}\right)=O\left(\frac{1}{d} \frac{d^{2}}{\sqrt{n}}\right)
$$

Then $\frac{1}{k}=O\left(n^{-2 \varepsilon} / d\right)$ and $\frac{|b|}{t}=o\left(n^{-\varepsilon} d^{-1}\right)$. Since now $|y| \leqslant y_{1}$, it is possible to replace $w$ by $t$ in the different $\exp (-k m w)$, in cost of an admissible error term:

Lemma 4.2. (i) We have

$$
\sum_{m=1}^{\infty} \frac{1}{m} \exp (-k m w)=\sum_{m=1}^{\infty} \frac{1}{m} \exp (-k m t)+O\left(\frac{|b|}{t}\right) .
$$

(ii) We have

$$
\begin{aligned}
\frac{1}{w} \sum_{m=1}^{\infty} \frac{1}{m^{2}} \exp (-k m w) & =\frac{1}{w} \sum_{m=1}^{\infty} \frac{1}{m^{2}} \exp (-k m t)-\frac{k i b}{w} \sum_{m=1}^{\infty} \frac{1}{m} \exp (-k m t) \\
& -\frac{k^{2} b^{2}}{2 w} \sum_{m=1}^{\infty} \exp (-k m t)+O\left(\frac{k^{3}|b|^{3}}{|w|}\right) \sum_{m=1}^{\infty} m \exp (-k m t)
\end{aligned}
$$

Proof. By standard approximations we have

$$
\begin{aligned}
\sum_{m=1}^{\infty} \frac{1}{m} \exp (-k m w) & =\sum_{m=1}^{\infty} \frac{1}{m}(1-(1-\exp (-k m i b))) \exp (-k m t) \\
& =\sum_{m=1}^{\infty} \frac{1}{m} \exp (-k m t)+\sum_{m=1}^{\infty} \frac{1}{m} \exp (-k m t) O(k m|b|) \\
& =\sum_{m=1}^{\infty} \frac{1}{m} \exp (-k m t)+O\left(\frac{k|b|}{e^{k t}-1}\right)=\sum_{m=1}^{\infty} \frac{1}{m} \exp (-k m t)+O\left(\frac{|b|}{t}\right) .
\end{aligned}
$$

This proves (i). Next we prove (ii). We have

$$
\begin{aligned}
\frac{1}{w} \sum_{m=1}^{\infty} \frac{1}{m^{2}} & \exp (-k m w)=\frac{1}{w} \sum_{m=1}^{\infty} \frac{1}{m^{2}} \exp (-k m t) \exp (-i k m b) \\
= & \frac{1}{w} \sum_{m=1}^{\infty} \frac{1}{m^{2}} \exp (-k m t)\left\{1-i k m b-\frac{(k m b)^{2}}{2}+O\left((k m|b|)^{3}\right)\right\}
\end{aligned}
$$

It remains to develop to end the proof of Lemma 4.2. 
We also have

$$
\sum_{m=1}^{\infty} m \exp (-k m t)=\frac{\exp (-k t)}{(1-\exp (-k t))^{2}}=\frac{1}{(\exp (k t)-1)(1-\exp (-k t))} \leqslant \frac{1}{(k t)^{2}},
$$

since for $u>0$,

$$
\mathrm{e}^{u}-1=u \sum_{n=0}^{\infty} \frac{u^{n}}{(n+1) !}>u \sum_{n=0}^{\infty} \frac{u^{n}}{n ! 2^{n}}=u \mathrm{e}^{\frac{u}{2}}
$$

thus $\left(1-\mathrm{e}^{-u}\right)>u \mathrm{e}^{-u / 2}$ and $\left(\mathrm{e}^{u}-1\right)\left(1-\mathrm{e}^{-u}\right)>u^{2}$.

This gives for $g_{k}(w)$ :

$$
\begin{aligned}
g_{k}(w) & =f(w) \exp \left\{-\frac{1}{w} \sum_{m=1}^{\infty} \frac{1}{m^{2}} \exp (-k m t)\right. \\
& +\frac{i k b}{w} \sum_{m=1}^{\infty} \frac{1}{m} \exp (-k m t)+\frac{k^{2} b^{2}}{2 w} \sum_{m=1}^{\infty} \exp (-k m t) \\
& \left.+\frac{1}{2} \sum_{m=1}^{\infty} \frac{1}{m} \exp (-k m t)+o\left(\frac{n^{-\varepsilon}}{d}\right)+O\left(k\left(\frac{|b|}{t}\right)^{3}\right)\right\} .
\end{aligned}
$$

The next step of the proof of Lemma 4.1 consists of "replacing" $\frac{1}{w}$ by $\frac{1}{t}$ and computing the terms arisen by this manipulation. We use the formula

$$
\frac{1}{w}=\frac{1}{t\left(1-\left(-i \frac{b}{t}\right)\right)}=\frac{1}{t}\left(1-i \frac{b}{t}-\frac{b^{2}}{t^{2}}+O\left(\frac{|b|^{3}}{t^{3}}\right)\right) .
$$

This gives for $g_{k}(w)$

$$
\begin{aligned}
g_{k}(w) & =f(w) \exp \left\{-\frac{1}{t} \sum_{m=1}^{\infty} \frac{1}{m^{2}} \exp (-k m t)+\frac{i b}{t^{2}} \sum_{m=1}^{\infty} \frac{1}{m^{2}} \exp (-k m t)\right. \\
& +\frac{b^{2}}{t^{3}} \sum_{m=1}^{\infty} \frac{1}{m^{2}} \exp (-k m t)+O\left(\frac{|b|^{3}}{t^{4}}\right)+\frac{i k b}{t} \sum_{m=1}^{\infty} \frac{1}{m} \exp (-k m t) \\
& +\frac{k b^{2}}{t^{2}} \sum_{m=1}^{\infty} \frac{1}{m} \exp (-k m t)+O\left(\frac{k|b|^{3}}{t^{3}} \log \left(1+\frac{1}{\mathrm{e}^{k t}-1}\right)\right) \\
& +\frac{k^{2} b^{2}}{2 t} \sum_{m=1}^{\infty} \exp (-k m t)+O\left(\frac{k^{2}|b|^{3}}{t^{2}\left(e^{k t}-1\right)}\right) \\
& \left.+\frac{1}{2} \sum_{m=1}^{\infty} \frac{1}{m} \exp (-k m t)+o\left(\frac{n^{-\varepsilon}}{d}\right)+O\left(\frac{k|b|^{3}}{t^{3}}\right)\right\} .
\end{aligned}
$$

We collect the terms with $i b$, the terms with $b^{2}$ :

$$
\begin{aligned}
g_{k}(w) & =f(w) \exp \left\{-\frac{1}{t} \sum_{m=1}^{\infty} \frac{1}{m^{2}} \exp (-k m t)+\frac{1}{2} \sum_{m=1}^{\infty} \frac{1}{m} \exp (-k m t)\right. \\
& +i b\left(\frac{1}{t^{2}} \sum_{m=1}^{\infty} \frac{1}{m^{2}} \exp (-k m t)+\frac{k}{t} \sum_{m=1}^{\infty} \frac{1}{m} \exp (-k m t)\right) \\
& +b^{2}\left(\frac{1}{t^{3}} \sum_{m=1}^{\infty} \frac{1}{m^{2}} \exp (-k m t)+\frac{k}{t^{2}} \sum_{m=1}^{\infty} \frac{1}{m} \exp (-k m t)+\frac{k^{2}}{2 t} \sum_{m=1}^{\infty} \exp (-k m t)\right) \\
& \left.+o\left(\frac{n^{-\varepsilon}}{d}\right)+O\left(\left(k+\frac{1}{t}\right) \frac{|b|^{3}}{t^{3}}\right)\right\} .
\end{aligned}
$$


Now we compute the different summations over $m$. By $(4 \cdot 1), \exp (-k m t)$ is close to $\exp \left(-k_{0} m t\right)$ if $m$ is not too large, but we have again some computations to do to control this approximation. For $s=0,1,2$ :

$$
\sum_{m=1}^{\infty} \frac{1}{m^{s}} \exp (-k m t)=\sum_{m=1}^{\infty} \frac{1}{m^{s}} \exp \left(-k_{0} m t\right) \exp \left(-\left(k-k_{0}\right) m t\right) .
$$

Since $\mathrm{e}^{x}=\sum_{n=0}^{M} \frac{x^{n}}{n !}+\sum_{n=M+1}^{\infty} \frac{x^{n}}{n !}$, we have

$$
\left|\mathrm{e}^{x}-\sum_{n=0}^{M} \frac{x^{n}}{n !}\right| \leqslant|x|^{M+1} \sum_{n=M+1}^{\infty} \frac{|x|^{n-M-1}}{n !} \leqslant|x|^{M+1} \mathrm{e}^{|x|} .
$$

Thus we obtain

$$
\begin{aligned}
\sum_{m=1}^{\infty} \frac{1}{m^{s}} \exp (-k m t) & =\sum_{m=1}^{\infty} \frac{1}{m^{s}} \exp \left(-k_{0} m t\right)\left\{1-\left(k-k_{0}\right) m t+\frac{1}{2}\left(k-k_{0}\right)^{2} m^{2} t^{2}\right. \\
& -\frac{1}{6}\left(k-k_{0}\right)^{3} m^{3} t^{3}+O\left(\left|k-k_{0}\right|^{4} m^{4} t^{4} \exp \left(\left|k-k_{0}\right| m t\right)\right) .
\end{aligned}
$$

By $(2 \cdot 2), \exp \left(\left|k-k_{0}\right| m t\right) \leqslant \exp \left(m k_{0} t / 2\right)$. Next we use the fact that $k_{0} t=\log 2$ :

$$
\begin{aligned}
\sum_{m=1}^{\infty} \frac{1}{m^{s}} \exp (-k m t) & =\sum_{m=1}^{\infty} \frac{1}{m^{s} 2^{m}}\left(1-\left(k-k_{0}\right) m t+\frac{1}{2}\left(k-k_{0}\right)^{2} m^{2} t^{2}\right. \\
& \left.-\frac{1}{6}\left(k-k_{0}\right)^{3} m^{3} t^{3}+O\left(\left|k-k_{0}\right|^{4} m^{4} t^{4} 2^{m / 2}\right)\right) \\
& =\sum_{m=1}^{\infty} \frac{1}{m^{s} 2^{m}}-\left(k-k_{0}\right) t \sum_{m=1}^{\infty} \frac{2^{-m}}{m^{s-1}} \\
& +\frac{1}{2}\left(k-k_{0}\right)^{2} t^{2} \sum_{m=1}^{\infty} \frac{2^{-m}}{m^{s-2}}-\frac{(k-k)^{3}}{6} t^{3} \sum_{m=1}^{\infty} \frac{2^{-m}}{m^{s-3}} \\
& +O\left(\left|k-k_{0}\right|^{4} t^{4} \sum_{m=1}^{\infty} \frac{2^{-m / 2}}{m^{s-4}}\right) .
\end{aligned}
$$

We obtain for the function $g_{k}$

$$
\begin{aligned}
g_{k}(w) & =f(w) \exp \left\{-\frac{1}{t} \sum_{m=1}^{\infty} \frac{1}{m^{2} 2^{m}}+\left(k-k_{0}\right) \sum_{m=1}^{\infty} \frac{1}{m 2^{m}}-\frac{\left(k-k_{0}\right)^{2} t}{2} \sum_{m=1}^{\infty} 2^{-m}\right. \\
& +O\left(\left|k-k_{0}\right|^{3} t^{2}\right)+\frac{1}{2} \sum_{m=1}^{\infty} \frac{1}{m 2^{m}}+O\left(\left|k-k_{0}\right| t\right) \\
& +i b\left(\frac{1}{t^{2}} \sum_{m=1}^{\infty} \frac{1}{m^{2} 2^{m}}-\frac{\left(k-k_{0}\right)}{t} \sum_{m=1}^{\infty} \frac{1}{m 2^{m}}+O\left(\left|k-k_{0}\right|^{2}\right)\right. \\
& \left.+\frac{k}{t} \sum_{m=1}^{\infty} \frac{1}{m 2^{m}}-k\left(k-k_{0}\right) \sum_{m=1}^{\infty} 2^{-m}+O\left(k t\left|k-k_{0}\right|^{2}\right)\right) \\
& +b^{2}\left(\frac{1}{t^{3}} \sum_{m=1}^{\infty} \frac{1}{m^{2} 2^{m}}+O\left(\frac{\left|k-k_{0}\right|}{t^{2}}\right)+\frac{k}{t^{2}} \sum_{m=1}^{\infty} \frac{1}{m 2^{m}}+O\left(\frac{k\left|k-k_{0}\right|}{t}\right)\right. \\
& \left.\left.+\frac{k^{2}}{2 t} \sum_{m=1}^{\infty} 2^{-m}+O\left(k^{2}\left|k-k_{0}\right|\right)\right)+o\left(n^{-\varepsilon} d^{-1}\right)+O\left(\left(k+\frac{1}{t}\right) \frac{|b|^{3}}{t^{3}}\right)\right\} .
\end{aligned}
$$


Next we compute the different sums on $m$ :

$$
\begin{aligned}
g_{k}(w) & =f(w) \exp \left\{-\frac{C_{2}}{t}+\left(k-k_{0}\right) \log 2-\frac{\left(k-k_{0}\right)^{2} t}{2}+\frac{1}{2} \log 2\right. \\
& +i b\left(\frac{C_{2}}{t^{2}}-\frac{\left(k-k_{0}\right)}{t} \log 2+\frac{k \log 2}{t}-k\left(k-k_{0}\right)\right)+b^{2}\left(\frac{C_{2}}{t^{3}}+\frac{k}{t^{2}} \log 2+\frac{k^{2}}{2 t}\right) \\
& +O\left(\left|k-k_{0}\right|^{3} t^{2}+\left|k-k_{0}\right| t+\left|k-k_{0}\right|^{2}|b|+\frac{\left|k-k_{0}\right| b^{2}}{t^{2}}\right) \\
& \left.+o\left(\frac{n^{-\varepsilon}}{d}\right)+O\left(\left(k+\frac{1}{t}\right) \frac{|b|^{3}}{t^{3}}\right)\right\} .
\end{aligned}
$$

Then by (4.1) the above error terms give $o\left(n^{-\varepsilon}|\mathcal{D}|^{-1}\right)$ and we can replace $-k\left(k-k_{0}\right)$ with $-k_{0}\left(k-k_{0}\right)$ in the coefficient of $i b$ and analogously in that of $b^{2}$. Finally,

$$
\begin{aligned}
g_{k}(w) & =f(w) \exp \left\{-\frac{C_{2}}{t}+\left(k-k_{0}\right) \log 2-\frac{\left(k-k_{0}\right)^{2} t}{2}+\frac{\log 2}{2}\right. \\
& \left.+i b\left(\frac{C_{2}}{t^{2}}+\frac{k_{0} \log 2}{t}-k_{0}\left(k-k_{0}\right)\right)+b^{2}\left(\frac{C_{2}}{t^{3}}+\frac{k_{0} \log 2}{t^{2}}+\frac{k_{0}^{2}}{2 t}\right)+o\left(\frac{n^{-\varepsilon}}{|\mathcal{D}|}\right)\right\},
\end{aligned}
$$

as claimed in Lemma 4.1.

\section{The term $S_{0}$, end of the proof of Theorem 1.1 in the case $\mathcal{D}=\{1, \ldots, \boldsymbol{d}\}$.}

As a special case of Lemma 4.1 applied with $\mathcal{D}=\{1, \ldots, d\}$, we remark that

$$
g_{k}\left(d x_{0}\right)=f\left(d x_{0}\right) \exp \left\{-\frac{C_{2}}{d x_{0}}+\left(k-k_{0}\right) \log 2-\frac{\left(k-k_{0}\right)^{2} d x_{0}}{2}+\frac{\log 2}{2}+o\left(\frac{n^{-\varepsilon}}{d}\right)\right\},
$$

and

$$
\begin{aligned}
\prod_{r=1}^{d} g_{N_{r}}\left(d x_{0}\right) & =f^{d}\left(d x_{0}\right) \exp \left\{-\frac{C_{2}}{x_{0}}+\sum_{r=1}^{d}\left(N_{r}-k_{0}\right) \log 2\right. \\
& \left.-\frac{d x_{0}}{2} \sum_{r=1}^{d}\left(N_{r}-k_{0}\right)^{2}+\frac{d \log 2}{2}+o\left(n^{-\varepsilon}\right)\right\} .
\end{aligned}
$$

Since $f(w)=\exp \left(\frac{\pi^{2}}{6 w}+\frac{1}{2} \log \frac{w}{2 \pi}+O(|w|)\right)$ for $w \rightarrow 0$ in $|\arg w| \leqslant \kappa<\pi / 2$ and $\Re e w>0$, we have for $|y| \leqslant y_{1} \leqslant n^{-\frac{3}{4}+\frac{\varepsilon}{3}}$ :

$$
\begin{aligned}
f\left(d\left(x_{0}+i y\right)\right) & =\exp \left(\frac{\pi^{2}}{6 d\left(x_{0}+i y\right)}+\frac{1}{2} \log \left(\frac{d\left(x_{0}+i y\right)}{2 \pi}\right)+O\left(d x_{0}\right)\right), \\
f^{d}\left(d\left(x_{0}+i y\right)\right) & =\exp \left(\frac{\pi^{2}}{6\left(x_{0}+i y\right)}+\frac{d}{2} \log \left(\frac{d\left(x_{0}+i y\right)}{2 \pi}\right)+O\left(d^{2} x_{0}\right)\right) \\
& =\exp \left(\frac{\pi^{2}}{6 x_{0}}\left(1-\frac{i y}{x_{0}}-\frac{y^{2}}{x_{0}^{2}}+O\left(\frac{|y|^{3}}{x_{0}^{3}}\right)\right)+\frac{d}{2} \log \left(\frac{d x_{0}}{2 \pi}\right)\right. \\
& \left.+\frac{d}{2} \log \left(1+\frac{i y}{x_{0}}\right)+O\left(d^{2} x_{0}\right)\right) \\
& =\exp \left(\frac{\pi^{2}}{6 x_{0}}-i y \frac{\pi^{2}}{6 x_{0}^{2}}-\frac{\pi^{2} y^{2}}{6 x_{0}^{3}}+\frac{d}{2} \log \left(\frac{d x_{0}}{2 \pi}\right)+o\left(n^{-\varepsilon}\right)\right) .
\end{aligned}
$$


We obtain for the integrand

$$
\begin{aligned}
P & :=\left\{\prod_{r=1}^{d} g_{N_{r}}\left(d\left(x_{0}+i y\right)\right)\right\} \exp \left((n-R-Q)\left(x_{0}+i y\right)\right) \\
& =\exp \left\{\frac{\pi^{2}}{6 x_{0}}-i y \frac{\pi^{2}}{6 x_{0}^{2}}-\frac{\pi^{2} y^{2}}{6 x_{0}^{3}}+\frac{d}{2} \log \left(\frac{d x_{0}}{2 \pi}\right)+o\left(n^{-\varepsilon}\right)\right. \\
& +(n-R-Q)\left(x_{0}+i y\right)-\frac{C_{2}}{x_{0}}+\sum_{r=1}^{d}\left(N_{r}-k_{0}\right) \log 2-\frac{1}{2} \sum_{r=1}^{d}\left(N_{r}-k_{0}\right)^{2} d x_{0}+\frac{d}{2} \log 2 \\
& +i d y\left(\frac{C_{2}}{d x_{0}^{2}}+\frac{k_{0} \log 2}{x_{0}}-k_{0} \sum_{r=1}^{d}\left(N_{r}-k_{0}\right)\right)+d^{2} y^{2}\left(\frac{C_{2}}{d^{2} x_{0}^{3}}+\frac{k_{0} \log 2}{d x_{0}^{2}}+\frac{k_{0}^{2}}{2 x_{0}}\right)+o\left(n^{-\varepsilon}\right\} .
\end{aligned}
$$

We collect terms in $i y, y^{2}$ :

$$
\begin{aligned}
P & =\exp \left\{\frac{\pi^{2}}{6 x_{0}}+\frac{d}{2} \log \left(\frac{d x_{0}}{2 \pi}\right)+(n-R-Q) x_{0}\right. \\
& -\frac{C_{2}}{x_{0}}+\sum_{r=1}^{d}\left(N_{r}-k_{0}\right) \log 2-\frac{1}{2} \sum_{r=1}^{d}\left(N_{r}-k_{0}\right)^{2} d x_{0}+\frac{d \log 2}{2} \\
& +i y\left(n-R-Q-\frac{\pi^{2}}{6 x_{0}^{2}}+\frac{C_{2}}{x_{0}^{2}}+\frac{d k_{0} \log 2}{x_{0}}-d k_{0} \sum_{r=1}^{d}\left(N_{r}-k_{0}\right)\right) \\
& \left.+y^{2}\left(-\frac{\pi^{2}}{6 x_{0}^{3}}+\frac{C_{2}}{x_{0}^{3}}+\frac{d k_{0} \log 2}{x_{0}^{2}}+\frac{d^{2} k_{0}^{2}}{2 x_{0}}\right)+o\left(n^{-\varepsilon}\right)\right\} .
\end{aligned}
$$

As a special case, we obtained that

$$
\begin{aligned}
\left\{\prod_{r=1}^{d} g_{N_{r}}\left(d x_{0}\right)\right\} \exp \left((n-R-Q) x_{0}\right) & =\exp \left\{\frac{\pi^{2}}{6 x_{0}}+\frac{d}{2} \log \left(\frac{d x_{0}}{2 \pi}\right)+(n-R-Q) x_{0}\right. \\
& -\frac{C_{2}}{x_{0}}+\sum_{r=1}^{d}\left(N_{r}-k_{0}\right) \log 2 \\
& \left.-\frac{1}{2} \sum_{r=1}^{d}\left(N_{r}-k_{0}\right)^{2} d x_{0}+\frac{d \log 2}{2}+o\left(n^{-\varepsilon}\right)\right\} .
\end{aligned}
$$

Consequently,

$$
\begin{aligned}
& \left\{\prod_{r=1}^{d} g_{N_{r}}\left(d\left(x_{0}+i y\right)\right)\right\} \exp \left((n-R-Q)\left(x_{0}+i y\right)\right) \\
& =\left\{\prod_{r=1}^{d} g_{N_{r}}\left(d x_{0}\right)\right\} \exp \left((n-R-Q) x_{0}\right) \\
& \times \exp \left\{i y\left(n-R-Q-\frac{\pi^{2}}{6 x_{0}^{2}}+\frac{1}{x_{0}^{2}} \sum_{m=1}^{\infty} \frac{1}{m^{2} 2^{m}}+\frac{d k_{0} \log 2}{x_{0}}-d k_{0} \sum_{r=1}^{d}\left(N_{r}-k_{0}\right)\right)\right. \\
& \left.+y^{2}\left(-\frac{\pi^{2}}{6 x_{0}^{3}}+\frac{1}{x_{0}^{3}} \sum_{m=1}^{\infty} \frac{1}{m^{2} 2^{m}}+\frac{d k_{0} \log 2}{x_{0}^{2}}+\frac{d^{2} k_{0}^{2}}{2 x_{0}}\right)+o\left(n^{-\varepsilon}\right)\right\} .
\end{aligned}
$$


The coefficient of $y^{2}$ in $(5 \cdot 3)$ is

$$
\frac{1}{x_{0}^{3}}\left(-\frac{\pi^{2}}{6}+\frac{\pi^{2}}{12}-\frac{\log ^{2} 2}{2}+d k_{0} x_{0} \log 2+\frac{d^{2} k_{0}^{2} x_{0}^{2}}{2}\right)=-\frac{2 \sqrt{3}}{\pi} n^{\frac{3}{2}}\left(1-\frac{12 \log ^{2} 2}{\pi^{2}}\right),
$$

where $\frac{12 \log ^{2} 2}{\pi^{2}}<\frac{12 \cdot 0.49}{\pi^{2}}<\frac{6}{\pi^{2}}<1$.

The coefficient of $i y$ in $(5 \cdot 3)$ is

$$
\begin{aligned}
& n-R-Q-\frac{1}{x_{0}^{2}}\left(\frac{\pi^{2}}{6}-\sum_{m=1}^{\infty} \frac{1}{m^{2} 2^{m}}-d k_{0} x_{0} \log 2\right)-d k_{0} \sum_{r=1}^{d}\left(N_{r}-k_{0}\right) \\
& =-R-Q+\frac{\log ^{2} 2}{2 x_{0}^{2}}-d k_{0} \sum_{r=1}^{d}\left(N_{r}-k_{0}\right)=-2 d k_{0} \sum_{r=1}^{d}\left(N_{r}-k_{0}\right)+O\left(n^{\frac{3}{4}-2 \varepsilon}\right) .
\end{aligned}
$$

Since $|y| O\left(n^{\frac{3}{4}-2 \varepsilon}\right)=o\left(n^{-\varepsilon}\right)$ we infer from $(5 \cdot 3)$ that

$$
\begin{aligned}
& \left\{\prod_{r=1}^{d} g_{N_{r}}\left(d\left(x_{0}+i y\right)\right)\right\} \exp \left((n-R-Q)\left(x_{0}+i y\right)\right) \\
& =\left\{\prod_{r=1}^{d} g_{N_{r}}\left(d x_{0}\right)\right\} \exp \left((n-R-Q) x_{0}\right) \\
& \times \exp \left\{-i y 2 d k_{0} \sum_{r=1}^{d}\left(N_{r}-k_{0}\right)-y^{2} \frac{2 \sqrt{3} n^{\frac{3}{2}}}{\pi}\left(1-\frac{12 \log ^{2} 2}{\pi^{2}}\right)+o\left(n^{-\varepsilon}\right)\right\} .
\end{aligned}
$$

Let $A=\frac{2 \sqrt{3} n^{\frac{3}{2}}}{\pi}\left(1-\frac{12 \log ^{2} 2}{\pi^{2}}\right),(A>0)$ and $B=2 d k_{0} \sum_{r=1}^{d}\left(N_{r}-k_{0}\right)$. Then, from $(5 \cdot 4)$

$$
\begin{aligned}
S_{0} & =\frac{d}{2 \pi} \int_{-y_{1}}^{y_{1}}\left\{\prod_{r=1}^{d} g_{N_{r}}\left(d\left(x_{0}+i y\right)\right)\right\} \exp \left((n-R-Q)\left(x_{0}+i y\right)\right) \mathrm{d} y \\
& =\frac{d}{2 \pi}\left\{\prod_{r=1}^{d} g_{N_{r}}\left(d x_{0}\right)\right\} \exp \left((n-R-Q) x_{0}\right) \int_{-y_{1}}^{y_{1}} \exp \left(-i y B-A y^{2}+o\left(n^{-\varepsilon}\right)\right) \mathrm{d} y .
\end{aligned}
$$

Lemma 5.1. We have :

$$
\int_{-y_{1}}^{y_{1}} \exp \left(-i y B-A y^{2}+o\left(n^{-\varepsilon}\right)\right) \mathrm{d} y=\sqrt{\frac{\pi}{A}} \exp \left(-\frac{B^{2}}{4 A}\right)\left\{1+o\left(n^{-\varepsilon}\right) \exp \left(\frac{B^{2}}{4 A}\right)\right\} .
$$

Proof. These are standard manipulations on Gaussian integrals thus we won't write all the details. Let $I_{A B}\left(y_{1}\right)$ be the integral of the left hand side of $(5 \cdot 5)$. Since for $|y| \leqslant y_{1}$, $\left.\exp \left(-i y B-A y^{2}+o\left(n^{-\varepsilon}\right)\right)=\left(1+o\left(n^{-\varepsilon}\right)\right) \exp \left(-i y B-A y^{2}\right)\right)$, we have :

$$
\begin{aligned}
I_{A B}\left(y_{1}\right) & =\int_{-\infty}^{+\infty} \exp \left(-i y B-A y^{2}\right) \mathrm{d} y \\
& +O\left(\int_{y_{1}}^{+\infty} \exp \left(-A y^{2}\right) \mathrm{d} y\right)+o\left(n^{-\varepsilon}\right) \int_{-\infty}^{+\infty} \exp \left(-A y^{2}\right) \mathrm{d} y .
\end{aligned}
$$

The main term is a Gaussian integral :

$$
\int_{-\infty}^{+\infty} \exp \left(-i y B-A y^{2}\right) \mathrm{d} y=\sqrt{\frac{\pi}{A}} \exp \left(-\frac{B^{2}}{4 A}\right) .
$$


For the error terms we have

$$
\int_{y_{1}}^{+\infty} \exp \left(-A y^{2}\right) \mathrm{d} y \ll \frac{1}{A y_{1}} \exp \left(-A y_{1}^{2}\right) \text { and } \int_{-\infty}^{+\infty} \exp \left(-A y^{2}\right) \ll \frac{1}{\sqrt{A}},
$$

the Lemma follows.

Furthermore

$$
\frac{B^{2}}{4 A}=O\left(n^{-\frac{3}{2}}\left(\sqrt{n} d \frac{n^{\frac{1}{4}} \sqrt{\log n}}{d w(n)}\right)^{2}\right)=o(\log n) .
$$

Thus the error term in Lemma 5.1 is :

$$
o\left(n^{-\varepsilon}\right) \exp \left(\frac{B^{2}}{4 A}\right)=o(1)
$$

Therefore

$$
S_{0}=(1+o(1)) \sqrt{\frac{\pi}{A}} \exp \left(-\frac{B^{2}}{4 A}\right) \frac{d}{2 \pi}\left\{\prod_{r=1}^{d} g_{N_{r}}\left(d x_{0}\right)\right\} \exp \left((n-R-Q) x_{0}\right) .
$$

Adding the estimates for the trivial parts (see $(2 \cdot 8),(3 \cdot 1)$ ) we obtain that for $n \equiv$ $R(\bmod d)$,

$$
\begin{aligned}
\Pi_{d}^{*}(n, \mathcal{R})= & \left\{\prod_{r=1}^{d} g_{N_{r}}\left(d x_{0}\right)\right\} \exp \left((n-R-Q) x_{0}\right) \\
& \times\left\{(1+o(1)) \sqrt{\frac{\pi}{A}} \exp \left(-\frac{B^{2}}{4 A}\right) \frac{d}{2 \pi}+O\left(\exp \left(-\frac{1+o(1)}{4 x_{0}}\right)\right)\right. \\
+ & \left.O\left(\exp \left(-\frac{\sqrt{3}}{\pi^{5} 3^{3}} n^{\frac{1}{4}+2 \varepsilon}\right)\right)+O\left(\exp \left(-\frac{\sqrt{3}}{\pi^{5} 3^{3}} n^{2 \varepsilon / 3}\right)\right)\right\} \\
= & (1+o(1)) \sqrt{\frac{\pi}{A}} \exp \left(-\frac{B^{2}}{4 A}\right) \frac{d}{2 \pi}\left\{\prod_{r=1}^{d} g_{N_{r}}\left(d x_{0}\right)\right\} \exp \left((n-R-Q) x_{0}\right) .
\end{aligned}
$$

To end the proof, it remains to insert the classical formula

$$
q(n)=(1+o(1)) \frac{1}{4.3^{1 / 4} n^{3 / 4}} \exp \left(\frac{\pi \sqrt{n}}{\sqrt{3}}\right),
$$

and our previous results on the $g_{N_{r}}\left(d x_{0}\right)$ (see $(5 \cdot 2)$ ), and to do the convenient computations. We obtain

$$
\begin{aligned}
\Pi_{d}^{*}(n, \mathcal{R}) & =(1+o(1)) 4.3^{1 / 4} q(n) \frac{1}{\sqrt{1-\frac{12(\log 2)^{2}}{\pi^{2}}}} \frac{d}{2 \sqrt{2} 3^{1 / 4}}\left(\frac{d}{2 \sqrt{3 n}}\right)^{d / 2} \exp \left(-\frac{B^{2}}{4 A}\right. \\
& \left.+\sum_{r=1}^{d}\left(N_{r}-k_{0}\right) \log 2-x_{0}\left(R+Q-\frac{\log ^{2} 2}{2 x_{0}^{2}}\right)-\frac{1}{2} \sum_{r=1}^{d}\left(N_{r}-k_{0}\right)^{2} d x_{0}\right) .
\end{aligned}
$$

By formulae (2.11) and (2.12) of [6] and by (2.4), we have:

$$
R+Q-\frac{\log ^{2} 2}{2 x_{0}^{2}}=\frac{d k_{0}}{2}+d k_{0} \sum_{r=1}^{d}\left(N_{r}-k_{0}\right)+\frac{d}{2} \sum_{r=1}^{d}\left(N_{r}-k_{0}\right)^{2}+o(\sqrt{n}) .
$$

Thus the argument of the exponential in (5.8) is

$$
\exp \left(-\frac{B^{2}}{4 A}+\ldots\right)=\exp \left(-\frac{B^{2}}{4 A}-\frac{\log 2}{2}-d x_{0} \sum_{r=1}^{d}\left(N_{r}-k_{0}\right)^{2}+o(1)\right) .
$$

Inserting this in (5.8) ends the proof of Theorem 1.1 for $\mathcal{D}=\{1, \ldots, d\}$. 


\section{First steps of the proof of Theorem 1.1 for $\mathcal{D} \neq\{1, \ldots, d\}$}

Like in Section 3 of [6], we apply Lemma 2.1 of [6], the Cauchy formula and write $z=x_{0}+i y$ :

$$
\begin{aligned}
\Pi_{d}^{*}\left(n, \mathcal{R}_{\mathcal{D}}\right)= & \frac{1}{2 \pi} \int_{-\pi}^{\pi}\left\{\prod_{r \in \mathcal{D}^{c}} h_{r}\left(x_{0}+i y\right)\right\} \\
& \times\left\{\prod_{r \in \mathcal{D}} g_{N_{r}}\left(d\left(x_{0}+i y\right)\right)\right\} \exp \left(\left(n-\mathcal{R}_{\mathcal{D}}-Q_{\mathcal{D}}\right)\left(x_{0}+i y\right)\right) \mathrm{d} y
\end{aligned}
$$

with

$$
h_{r}(z)=\prod_{j=0}^{\infty}(1+\exp (-(r+j d) z)) .
$$

When $\mathcal{D}^{c} \neq \varnothing$ and $\mathcal{D}^{c} \neq\{d\}$, the functions $h_{r}$ are not $2 \pi / d$-periodic but we still split the integral in intervales of length $2 \pi / d$ in order to use our previous work on the functions $g_{k}$.

$$
\Pi_{d}^{*}\left(n, \mathcal{R}_{\mathcal{D}}\right)=\frac{1}{2 \pi} \sum_{|\lambda| \leqslant\left\lfloor\frac{d-1}{2}\right\rfloor} \int_{-\frac{\pi}{d}+\frac{2 \lambda \pi}{d}}^{\frac{\pi}{d}+\frac{2 \lambda \pi}{d}} \ldots+B
$$

with

$$
B= \begin{cases}0 & \text { if } d \text { is odd } \\ \int_{-\pi}^{-\pi+\frac{\pi}{d}} \ldots+\int_{\pi-\frac{\pi}{d}}^{\pi} \ldots=\int_{\pi-\frac{\pi}{d}}^{\pi+\frac{\pi}{d}} & \text { if } d \text { is even. }\end{cases}
$$

Next we do some convenient change of variables :

$$
\begin{aligned}
\Pi_{d}^{*}\left(n, \mathcal{R}_{\mathcal{D}}\right)= & \frac{1}{2 \pi} \sum_{-\frac{d}{2}<\lambda \leqslant \frac{d}{2}} \int_{-\frac{\pi}{d}}^{\frac{\pi}{d}}\left\{\prod_{r \in \mathcal{D}^{c}} h_{r}\left(x_{0}+i y+i \frac{2 \lambda \pi}{d}\right)\right\}\left\{\prod_{r \in \mathcal{D}} g_{N_{r}}\left(d\left(x_{0}+i y\right)\right)\right\} \\
& \times \exp \left(\left(n-\mathcal{R}_{\mathcal{D}}-Q_{\mathcal{D}}\right)\left(x_{0}+i y\right)+\left(n-\mathcal{R}_{\mathcal{D}}\right) \frac{2 i \lambda \pi}{d}\right) \mathrm{d} y \\
& =\sum_{-\frac{d}{2}<\lambda \leqslant \frac{d}{2}} S(\lambda),
\end{aligned}
$$

say. We will write the splitting

$$
S(\lambda)=S_{0}(\lambda)+S_{1}(\lambda)+S_{2}(\lambda)
$$

where in $S_{0}(\lambda)$ the range of integration for $y$ is $|y| \leqslant y_{1}$, in $S_{1}(\lambda)$ it is for $y_{1} \leqslant|y| \leqslant y_{2}$ (with $y_{2}=3 \pi x_{0}$ ) and in $S_{2}(\lambda)$ we take $y_{2} \leqslant|y| \leqslant \frac{\pi}{d}$ (cf. (4.11) of $[6]$ ).

\section{Upper bounds of $S_{1}(\lambda)$ and $S_{2}(\lambda)$}

To obtain a convenient upper bound of these terms we first remark that

$$
\left|h_{r}\left(x_{0}+i y+\frac{2 i \pi \lambda}{d}\right)\right| \leqslant h_{r}\left(x_{0}\right) .
$$

Let $j \in \mathbb{N}$. To prove $(7 \cdot 1)$ it is enough to prove that each $T_{j} \leqslant 1$ with

$$
T_{j}:=\frac{\left|1+\exp \left(-(r+j d)\left(x_{0}+i y+\frac{2 i \pi \lambda}{d}\right)\right)\right|}{\left|1+\exp \left(-(r+j d) x_{0}\right)\right|} .
$$


By a simple computation we have

$$
T_{j}^{2}=1-\frac{4 \exp \left(-x_{0}(r+j d)\right) \sin ^{2}\left(\frac{y}{2}(r+j d)+\frac{\pi \lambda r}{d}\right)}{\left(1+\exp \left(-x_{0}(r+j d)\right)\right)^{2}} \leqslant 1 .
$$

By Lemma 2.1 we have

$$
\left|S_{2}(\lambda)\right| \leqslant\left\{\prod_{r \in \mathcal{D}} g_{N_{r}}\left(d x_{0}\right)\right\}\left\{\prod_{r \in \mathcal{D}^{c}} h_{r}\left(x_{0}\right)\right\} \exp \left(\left(n-R_{\mathcal{D}}-Q_{\mathcal{D}}\right) x_{0}\right) \exp \left(\frac{|\mathcal{D}|(-1+o(1))}{4 d x_{0}}\right) .
$$

By Lemma 3.1 we also have

$$
\left|S_{1}(\lambda)\right| \leqslant\left\{\prod_{r \in \mathcal{D}} g_{N_{r}}\left(d x_{0}\right)\right\}\left\{\prod_{r \in \mathcal{D}^{c}} h_{r}\left(x_{0}\right)\right\} \exp \left(\left(n-R_{\mathcal{D}}-Q_{\mathcal{D}}\right) x_{0}\right) \exp \left(-\frac{|\mathcal{D}| \sqrt{3} n^{2 \varepsilon / 3}}{27 \pi^{5} d}\right) .
$$

If $|\mathcal{D}|$ is small, i.e., if $|\mathcal{D}| \leqslant d n^{-\varepsilon / 3}$ this last estimate for $S_{1}(\lambda)$ is not sufficient. However, by Lemma 3.1 (i), the contribution of the range $n^{-5 / 8+\varepsilon} \leqslant|y| \leqslant 3 \pi x_{0}$ to $S_{1}(\lambda)$ is

$$
\leqslant\left\{\prod_{r \in \mathcal{D}} g_{N_{r}}\left(d x_{0}\right)\right\}\left\{\prod_{r \in \mathcal{D}^{c}} h_{r}\left(x_{0}\right)\right\} \exp \left(\left(n-R_{\mathcal{D}}-Q_{\mathcal{D}}\right) x_{0}\right) \exp \left(-\frac{|\mathcal{D}| \sqrt{3} n^{1 / 4+2 \varepsilon}}{27 \pi^{5} d}\right) .
$$

Thus it remains to handle the case $|\mathcal{D}| \leqslant d n^{-\varepsilon / 3}$ in the range $y_{1} \leqslant|y|<n^{-5 / 8+\varepsilon}$.

- First we study the case $\lambda=0$. We use a similar argument as in the proof of Lemma 3.1.

For any $1 \leqslant r \leqslant d$, let $J_{r}$ denote the set of the integers $j$ such that

$$
\frac{1}{2 x_{0}} \leqslant r+j d \leqslant \frac{1}{x_{0}}
$$

Then for $j \in J_{r}$ we have

$$
\frac{\exp \left(-x_{0}(r+j d)\right)}{\left(1+\exp \left(-x_{0}(r+j d)\right)\right)^{2}} \geqslant \frac{1}{\mathrm{e}\left(1+\mathrm{e}^{-1 / 2}\right)^{2}}
$$

This gives for the correspondent $T_{j}$ :

$$
T_{j}^{2} \leqslant 1-\frac{4}{\mathrm{e}\left(1+\mathrm{e}^{-1 / 2}\right)^{2}} \sin ^{2}\left(\frac{y}{2}(r+j d)\right) .
$$

Next, quite like in the proof of Lemma 3.1, we have for $n$ large enough

$$
\begin{aligned}
\left|h_{r}\left(x_{0}+i y\right)\right| & \leqslant h_{r}\left(x_{0}\right)\left(1-\frac{4}{\mathrm{e}\left(1+\mathrm{e}^{-1 / 2}\right)^{2}} \frac{y_{1}^{2}}{4 \pi^{2} x_{0}^{2}}\right)^{\left|J_{r}\right| / 2} \\
& \leqslant h_{r}\left(x_{0}\right) \exp \left(-\frac{y_{1}^{2}}{48 \pi^{2} d x_{0}^{3}}\right) \leqslant h_{r}\left(x_{0}\right) \exp \left(-\frac{\sqrt{3} n^{2 \varepsilon / 3}}{2 \pi^{5} d}\right) .
\end{aligned}
$$

This upper bound combined with Lemma 3.1 is sufficient to obtain

$$
\left|S_{1}(0)\right| \leqslant\left\{\prod_{r \in \mathcal{D}} g_{N_{r}}\left(d x_{0}\right)\right\}\left\{\prod_{r \in \mathcal{D}^{c}} h_{r}\left(x_{0}\right)\right\} \exp \left(\left(n-R_{\mathcal{D}}-Q_{\mathcal{D}}\right) x_{0}\right) \exp \left(-\frac{\sqrt{3} n^{2 \varepsilon / 3}}{27 \pi^{5}}\right) .
$$

- Now we suppose that $\lambda \neq 0$. We write $\frac{\lambda}{d}=\frac{\lambda^{\prime}}{d^{\prime}}$ with $\left(\lambda^{\prime}, d^{\prime}\right)=1$ and $d^{\prime}>0$. 
First we suppose that $d^{\prime} \geqslant n^{\varepsilon / 4}$. Since $\left(\lambda^{\prime}, d^{\prime}\right)=1$, there are $\frac{d^{\prime}}{4}+O(1)$ integers $r_{0} \in\left\{1, \ldots, d^{\prime}\right\}$ such that $\frac{\lambda^{\prime} r_{0}}{d^{\prime}} \bmod 1 \in\left[\frac{1}{4}, \frac{1}{2}\right]$. Thus there are $\frac{d}{4}+O\left(\frac{d}{d^{\prime}}\right)$ integers $r \in\{1, \ldots, d\}$ such that $\frac{\lambda^{\prime} r}{d^{\prime}} \bmod 1 \in\left[\frac{1}{4}, \frac{1}{2}\right]$ (again for $n$ large enough).

Since $|\mathcal{D}| \leqslant d n^{-\varepsilon / 3}<\frac{d}{4}+O\left(\frac{d}{d^{\prime}}\right)$ for $n$ large enough, there exists $r_{1} \in \mathcal{D}^{c}$ such that $\frac{\lambda^{\prime} r_{1}}{d^{\prime}}$ $\bmod 1 \in\left[\frac{1}{4}, \frac{1}{2}\right]$. For $j \in J_{r_{1}},\left|\left(r_{1}+j d\right) y\right| \ll n^{-1 / 8+\varepsilon}$. Thus $\left.\sin ^{2}\left(\left(r_{1}+j d\right) \frac{y}{2}+\frac{\lambda r_{1} \pi}{d}\right)\right) \geqslant$ $\sin ^{2} \frac{\pi}{6}=\frac{1}{4}$.

This gives

$$
\prod_{j \in J_{r_{1}}}\left|T_{j}\left(r_{1}\right)\right|^{2} \leqslant\left(\frac{11}{12}\right)^{\left|J_{r_{1}}\right|} \leqslant \exp \left(-\frac{1}{30 d x_{0}}\right),
$$

which is a sufficient upper bound.

Now we suppose that $2 \leqslant d^{\prime}<n^{\varepsilon / 4}$. There are $d-\frac{d}{d^{\prime}}$ integers $r$ such that $d^{\prime} \not$. For these integers $r$ and $j \in J_{r}$, we have

$$
\sin ^{2}\left((r+j d) \frac{y}{2}+\frac{\pi \lambda r}{d}\right) \geqslant \sin ^{2}\left(\frac{\pi}{2 d^{\prime}}\right) \geqslant \frac{1}{d^{2}} .
$$

Since $|\mathcal{D}| \leqslant d n^{-\varepsilon / 3}$, there are at least $d / 3$ such integers $r \in \mathcal{D}^{c}$ such that $d^{\prime} \chi_{r}$.

Thus we have :

$$
\prod_{r \in \mathcal{D}^{c}} \frac{\left|h_{r}\left(x_{0}+i y+\frac{2 i \pi \lambda}{d}\right)\right|}{h_{r}\left(x_{0}\right)} \leqslant \prod_{\substack{r \in \mathcal{D}^{c} \\ r \neq 0\left(\bmod d^{\prime}\right)}} \prod_{j \in J_{r}}\left(1-\frac{1}{6 d^{2}}\right) \leqslant \exp \left(-\frac{d}{48 d^{3} x_{0}}\right),
$$

which is a sufficient upper bound when $d \leqslant n^{1 / 4-2 \varepsilon}$.

\section{The terms $S_{0}(\lambda)$ for $\lambda \neq 0$}

We have to consider the integrals

$$
\begin{aligned}
S_{0}(\lambda) & =\frac{1}{2 \pi} \int_{|y| \leqslant y_{1}}\left\{\prod_{r \in \mathcal{D}^{c}} h_{r}\left(x_{0}+i y+\frac{2 i \pi \lambda}{d}\right)\right\}\left\{\prod_{r \in \mathcal{D}} g_{N_{r}}\left(d\left(x_{0}+i y\right)\right)\right\} \\
& \times \exp \left(\left(n-R_{\mathcal{D}}-Q_{\mathcal{D}}\right)\left(x_{0}+i y+\frac{2 i \pi \lambda}{d}\right)\right) \mathrm{d} y .
\end{aligned}
$$

- First we suppose that there exists $r \in \mathcal{D}^{c}$ such that $\lambda r \not \equiv 0(\bmod d)$. In the previous section we remarked that

$$
\left|h_{r}\left(x_{0}+i y+\frac{2 i \pi \lambda}{d}\right)\right|=h_{r}\left(x_{0}\right) \prod_{j=0}^{\infty}\left(1-\frac{4 \exp \left(-x_{0}(r+j d)\right) \sin ^{2}\left(\frac{y}{2}(r+j d)+\frac{\pi \lambda r}{d}\right)}{\left(1+\exp \left(-x_{0}(r+j d)\right)^{2}\right.}\right)^{1 / 2} .
$$

We work again with the sets $J_{r}$ defined by $(7 \cdot 2)$ in the previous section. For $j \in J_{r}$, $\lambda r \equiv a(\bmod d), 1 \leqslant|a| \leqslant d / 2$ and $|y| \leqslant y_{1}$ we have:

$\sin ^{2}\left(\frac{y}{2}(r+j d)+\frac{\pi \lambda r}{d}\right)=\sin ^{2}\left(\frac{\pi|a|}{d} \pm \frac{|y|}{2}(r+j d)\right) \geqslant \sin ^{2}\left(\frac{\pi|a|}{d}-\frac{y_{1}}{x_{0}}\right) \geqslant \sin ^{2}\left(\frac{\pi}{d}-\frac{y_{1}}{x_{0}}\right) \geqslant \frac{3}{d^{2}}$,

for $n$ large enough (we recall that $d \leqslant n^{1 / 4-2 \varepsilon}$ ).

Since $\left|J_{r}\right|=\frac{1}{2 d x_{0}}+O(1) \geqslant \frac{1}{3 d x_{0}}$, we have:

$$
\begin{aligned}
\left|h_{r}\left(x_{0}+i y+\frac{2 i \pi \lambda}{d}\right)\right| & \leqslant h_{r}\left(x_{0}\right) \prod_{j \in J_{r}}\left(1-\frac{12}{\mathrm{e} d^{2}\left(1+\mathrm{e}^{-1 / 2}\right)^{2}}\right)^{1 / 2} \\
& \leqslant h_{r}\left(x_{0}\right) \exp \left(\frac{1}{6 d x_{0}} \log \left(1-\frac{12}{\mathrm{e} d^{2}\left(1+\mathrm{e}^{-1 / 2}\right)^{2}}\right)\right) \\
& \leqslant h_{r}\left(x_{0}\right) \exp \left(-\frac{2}{\mathrm{e} d^{3} x_{0}\left(1+\mathrm{e}^{-1 / 2}\right)^{2}}\right) .
\end{aligned}
$$


Thus if there exists $r_{0} \in \mathcal{D}^{c}$ such that $\lambda r_{0} \not \equiv 0(\bmod d)$ then

$$
\left|S_{0}(\lambda)\right| \leqslant \prod_{r \in \mathcal{D}} g_{N_{r}}\left(d x_{0}\right) \prod_{r \in \mathcal{D}^{c}} h_{r}\left(x_{0}\right) \exp \left(\left(n-R_{\mathcal{D}}-Q_{\mathcal{D}}\right) x_{0}\right) \exp \left(-\frac{2}{\mathrm{e} d^{3} x_{0}\left(1+\mathrm{e}^{-1 / 2}\right)^{2}}\right) .
$$

This upper bound is sufficient only for $d \leqslant n^{1 / 6-\varepsilon}$.

- We suppose now that

$$
\lambda r \equiv 0(\bmod d) \text { for all } r \in D^{c} .
$$

If $\mathcal{D}^{c}=\{d\}$ then by $(1 \cdot 1), n-R_{D}-Q_{\mathcal{D}} \equiv 0(\bmod d)$ and $S_{0}(\lambda)=S_{0}(0)$ for all $\lambda$.

In the general case, if (8.2) holds then we must have $\frac{d}{(\lambda, d)} \mid r$. Thus $\frac{d}{(\lambda, d)} \mid \delta$ and again by $(1 \cdot 1)$, we have $n-R_{\mathcal{D}}-Q_{\mathcal{D}} \equiv 0\left(\bmod \frac{d}{(\lambda, d)}\right) . T h u s \exp \left(\left(n-R_{\mathcal{D}}-Q_{\mathcal{D}}\right) \frac{2 i \pi \lambda}{d}\right)=1$ and $S_{0}(\lambda)=S_{0}(0)$.

For $\mathcal{D}^{c}$ given, there exists $\delta$ integers $\lambda$ modulo $d$ such that $r \lambda \equiv 0(\bmod d)$ for all $r \in \mathcal{D}^{c}$.

We summarize these observations in the following lemma.

Lemma 8.1. For $d \leqslant n^{1 / 6-\varepsilon}$ we have:

$$
\sum_{-\frac{d}{2}<\lambda \leqslant \frac{d}{2}} S_{0}(\lambda)=\delta S_{0}(0)(1+o(1))
$$

\section{The function $h_{r}^{-1}$ in the range $|y| \leqslant y_{1}$}

The generating function associated to unequal partitions is $h(z)=\prod_{j=1}^{\infty}\left(1+z^{j}\right)$. For $S_{0}(0)$ it remains to handle the integral

$$
\frac{1}{2 \pi} \int_{|y| \leqslant y_{1}} h\left(\exp \left(-\left(x_{0}+i y\right)\right)\right)\left\{\prod_{r \in \mathcal{D}} \frac{g_{N_{r}}\left(d\left(x_{0}+i y\right)\right)}{h_{r}\left(x_{0}+i y\right)}\right\} \exp \left(\left(n-R_{\mathcal{D}}-Q_{\mathcal{D}}\right)\left(x_{0}+i y\right)\right) \mathrm{d} y .
$$

In this section we will state an asymptotic estimate related to $h_{r}^{-1}$ in the range $|y| \leqslant y_{1}$.

The following method looks like the general method of Meinardus [7] for studying generating functions associated to partitions (this method is presented in details in the book of Andrews [1]). In fact we were also inspired by the chapter on the application of saddle point method to the partitions function in the Master course of Tenenbaum [8].

In Lemma 4.1, we obtained an estimation of $g_{k}(d w)$ in function of $f(d w)$. This leads us to try in fact to obtain an estimation of $U_{r}(z):=f(d z) h_{r}^{-1}(z)$ for $|y| \leqslant y_{1}$ instead of $h_{r}^{-1}(z)$. This change will make some computations easier. Thus we consider

$$
U_{r}(z)=\prod_{j=1}^{\infty}(1-\exp (-j d z))^{-1} \prod_{j=0}^{\infty}(1+\exp (-(r+j d) z))^{-1} .
$$

The main result of this section is the following lemma

Lemma 9.1. Let $\eta>0$. For $|y| \leqslant y_{1}$ and $1 \leqslant r \leqslant d$, we have

$$
U_{r}(z)=\exp \left(\frac{\pi^{2}}{12 d z}+\left(\frac{r}{d}-1\right) \log 2+\frac{1}{2} \log \left(\frac{d z}{\pi}\right)+O\left(d^{1+\eta} r^{-\eta}|z|\right)+O\left(d^{-1} n^{-2 \varepsilon}\right)\right) .
$$

In the next section we will apply this lemma with $\eta>0$ small enough such that $d^{2+\eta}|z| \leqslant n^{-\varepsilon}$. 
Proof. If $r=d$, there are quite no work to do since

$$
U_{d}(z)=\prod_{j=1}^{\infty}(1+\exp (-j d z))^{-1}(1-\exp (-j d z))^{-1}=\prod_{j=1}^{\infty}(1-\exp (-2 j d z))^{-1}=f(2 d z) .
$$

Thus

$$
U_{d}(z)=\exp \left(\frac{\pi^{2}}{12 d z}+\frac{1}{2} \log \left(\frac{2 d z}{2 \pi}\right)+O(d|z|)\right) .
$$

Now we suppose that $r \neq d$. We prefer to work with

$$
\begin{aligned}
\tilde{U}_{r}(z) & :=\prod_{j=1}^{\infty}(1-\exp (-j d z))^{-1}(1+\exp (-(r+j d) z))^{-1} \\
& =U_{r}(z)(1+\exp (-r z))=U_{r}(z) u_{r}(z) .
\end{aligned}
$$

We easily see that

$$
u_{r}(z)=(2+O(r|z|))
$$

Let $F(v, s)=\sum_{k=1}^{\infty} \frac{\exp (-k v)}{k^{s}}$. If $\Re e v>0$, then $s \mapsto F(v, s)$ is analytic on $\mathbb{C}$.

The first step of the proof is the following result.

Lemma 9.2. For $r \neq d, \eta>0, z=x_{0}+i y$ with $|y| \leqslant y_{1}$, we have

$$
\begin{aligned}
\log \left(\tilde{U}_{r}(z)\right) & =\frac{1}{d z}\left(\frac{\pi^{2}}{6}+F(r z+i \pi, 2)\right)+\frac{1}{2} \log \left(\frac{d z}{2 \pi}\right) \\
& -\frac{1}{2} F(r z+i \pi, 1)+\frac{d z}{12}\left(-\frac{1}{2}+F(z+i \pi, 0)\right)+O\left(|z| r^{-\eta} d^{1+\eta}\right) .
\end{aligned}
$$

The last term $\frac{d z}{12}\left(-\frac{1}{2}+F(z+i \pi, 0)\right)$ in the above formula could be removed because it is $O\left(|z| r^{-\eta} d^{1+\eta}\right)$. The following proof uses Mellin formula and looks like the general method of Meinardus for studying generating functions associated to partitions.

We begin by some standard manipulations

$$
\begin{aligned}
\tilde{U}_{r}(z) & =\exp \left\{-\sum_{j=1}^{\infty}(\log (1+\exp (-(r+j d) z))+\log (1-\exp (-j d z))\}\right. \\
& =\exp \left\{\sum_{j, k \geqslant 1} \frac{\exp (-j k d z)}{k}\left((-1)^{k} \exp (-k r z)+1\right)\right\} .
\end{aligned}
$$

Let us write

$$
\log \left(\tilde{U}_{r}(z)\right)=\sum_{m=1}^{\infty} \frac{\beta(m)}{m} \exp (-m d z)
$$

with

$$
\beta(m)=\sum_{j k=m} j\left((-1)^{k} \exp (-k r z)+1\right) .
$$

By the Mellin transform formula we have:

$$
\log \left(\tilde{U}_{r}(z)\right)=\sum_{m \geqslant 1} \frac{\beta(m)}{m} \cdot \frac{1}{2 i \pi} \int_{2-i \infty}^{2+i \infty} \frac{\Gamma(s)}{(m d z)^{s}} \mathrm{~d} s
$$


But the Dirichlet series is

$$
\begin{aligned}
\sum_{m \geqslant 1} \frac{\beta(m)}{m^{s+1}} & =\sum_{j \geqslant 1} \frac{1}{j^{s}} \sum_{k \geqslant 1} \frac{\left(1+(-1)^{k} \exp (-k r z)\right)}{k^{s+1}} \\
& =\zeta(s)(\zeta(s+1)+F(r z+i \pi, s+1)) .
\end{aligned}
$$

Thus we have

$$
\log \left(\tilde{U}_{r}(z)\right)=\frac{1}{2 i \pi} \int_{2-i \infty}^{2+i \infty} \frac{\Gamma(s) \zeta(s)(\zeta(s+1)+F(r z+i \pi, s+1))}{(d z)^{s}} \mathrm{~d} s .
$$

Let $\eta \in] 0,1[$. We move the integral until the line $\Re e s=-1-\eta$. This gives

$$
\begin{aligned}
\log \left(\tilde{U}_{r}(z)\right) & =\frac{1}{2 i \pi} \int_{-1-\eta-i \infty}^{-1-\eta+i \infty} \frac{\Gamma(s) \zeta(s)(\zeta(s+1)+F(r z+i \pi, s+1))}{(d z)^{s}} \mathrm{~d} s \\
& +\operatorname{Res}(1)+\operatorname{Res}(0)+\operatorname{Res}(-1)+E,
\end{aligned}
$$

where $E$ is the error term arising from the horizontal branches.

For $\Re$ e $s \in[-1-\eta, 2]$ we have:

$$
\begin{aligned}
F(z r+i \pi, s+1) & =\sum_{\ell \geqslant 1} \mathrm{e}^{-2 \ell z r}\left(\frac{1}{(2 \ell)^{s+1}}-\frac{\mathrm{e}^{r z}}{(2 \ell-1)^{s+1}}\right) \\
& =\sum_{\ell \geqslant 1} \mathrm{e}^{-2 \ell z r}\left(\frac{1}{(2 \ell)^{s+1}}-\frac{1}{(2 \ell-1)^{s+1}}\right)+O\left(r|z| \sum_{\ell \geqslant 1} \frac{\mathrm{e}^{-2 \ell r x_{0}}}{\ell^{1+\Re e s}}\right) .
\end{aligned}
$$

When $\ell$ is small, the term $\mathrm{e}^{-\ell r x_{0}}$ is $O(1)$. Thus for $-1-\eta \leqslant \Re e s \leqslant 2$, we have :

$$
\sum_{\ell \geqslant 1} \frac{\mathrm{e}^{-2 \ell r x_{0}}}{\ell^{1+\Re e s}} \ll \sum_{\ell \leqslant 100 / r x_{0}} \ell^{\eta}+\sum_{\ell>100 / r x_{0}} \ell^{\eta} \mathrm{e}^{-2 \ell r x_{0}} \ll\left(r x_{0}\right)^{-1-\eta},
$$

since for the second sum we have

$$
\sum_{\ell>100 / r x_{0}} \ell^{\eta} \mathrm{e}^{-2 \ell r x_{0}} \ll \sum_{\ell>100 / r x_{0}} \ell \mathrm{e}^{-2 \ell r x_{0}}\left(r x_{0}\right)^{1-\eta} \ll \frac{\left(r x_{0}\right)^{1-\eta}}{\left(1-\exp \left(-2 r x_{0}\right)\right)^{2}} .
$$

For the other term we have

$$
\left|\sum_{\ell \geqslant 1} \mathrm{e}^{-2 \ell r z}\left(\frac{1}{(2 \ell)^{1+s}}-\frac{1}{(2 \ell-1)^{1+s}}\right)\right| \ll(1+|s|) \sum_{\ell \geqslant 1} \frac{\mathrm{e}^{-2 \ell r x_{0}}}{\ell^{1-\eta}} \ll \frac{1+|s|}{\left(r x_{0}\right)^{\eta}} .
$$

Thus for $-1-\eta \leqslant \Re e s \leqslant 2$, we have

$$
|F(z r+i \pi, s+1)| \ll|s|\left(r x_{0}\right)^{-\eta}+r|z|\left(r x_{0}\right)^{-1-\eta} .
$$

We will also use the following classical results for the functions $\zeta$ and $\Gamma$ in vertical strips:

- there exists $H>0$ such that $|\zeta(s)| \ll|\Im m s|^{H}$ for $-3 \leqslant \Re e s \leqslant 3$ (in fact more generally for $\Re e s \in\left[\sigma_{1}, \sigma_{2}\right]$ ) and $|\Im m s| \geqslant 1$ (cf. [2] Theorem 12.23 p. 270 for a more precise formulation) ; 
- for $-3 \leqslant \Re e s \leqslant 3$ (or $\Re e s \in\left[\sigma_{1}, \sigma_{2}\right]$ ) and $|\Im m s| \rightarrow+\infty$, ([9] Corollaire II.0.13 p. 182) we have

$$
\Gamma(s)=\left(1+O\left(|\Im m s|^{-1}\right)\right) \sqrt{2 \pi}|\Im m s|^{\Re e s-\frac{1}{2}} \mathrm{e}^{-\pi|\Im m s| / 2} \mathrm{e}^{i \alpha(s)},
$$

with $\alpha(s)=(\Im m s) \log |\Im m s|-\Im m s+\frac{1}{2} \pi\left(\Re e s-\frac{1}{2}\right) \operatorname{sgn}(\Im m s)$.

With this two formulae and by $(9 \cdot 2)$ we easily see that

$$
\lim _{T \rightarrow+\infty} \int_{-1-\eta \pm i T}^{2 \pm i T} \frac{\Gamma(s) \zeta(s)(\zeta(s+1)+F(r z+i \pi, s+1))}{(d z)^{s}} \mathrm{~d} s=0,
$$

and

$$
\left|\int_{\Re e s=-1-\eta} \frac{\Gamma(s) \zeta(s)(\zeta(s+1)+F(r z+i \pi, s+1))}{(d z)^{s}} \mathrm{~d} s\right| \ll r^{-\eta} d^{1+\eta}|z| .
$$

Now we compute the different residues:

We have

$$
\operatorname{Res}(1)=\frac{\Gamma(1)(\zeta(2)+F(r z+i \pi, 2))}{d z}=\frac{1}{d z}\left(\frac{\pi^{2}}{6}+F(r z+i \pi, 2)\right) .
$$

In $s=0$, we have two poles, one from $\Gamma$, the other from the function $s \mapsto \zeta(s+1)$. We use the well known results $\left.\Gamma^{\prime}(1)=-\gamma, \zeta(s)=\frac{1}{s-1}+\gamma+O(|s-1|)\right)$.

Thus $\operatorname{Res}(0)$ is the coefficient in $s^{-1}$ in the following formula:

$$
\begin{aligned}
& \left(\frac{1-\gamma s+O\left(|s|^{2}\right)}{s}\right)\left(\zeta(0)+s \zeta^{\prime}(0)+O\left(|s|^{2}\right)\right) \\
& \times\left[\frac{1}{s}+\gamma+O(|s|)+F(r z+i \pi, 1)\right]\left(1-s \log (d z)+O\left(|s|^{2}\right)\right) .
\end{aligned}
$$

Since $\zeta(0)=-\frac{1}{2}, \zeta^{\prime}(0)=-\frac{1}{2} \log (2 \pi)$, we find

$$
\operatorname{Res}(0)=\frac{1}{2}\left(\log \left(\frac{d z}{2 \pi}\right)-F(r z+i \pi, 1)\right) .
$$

In $s=-1, \Gamma$ has a simple pole with residue -1 thus we have:

$$
\operatorname{Res}(-1)=-\zeta(-1)(\zeta(0)+F(r z+i \pi, 0)) d z .
$$

Since $\zeta(-1)=-\frac{1}{12}$, we obtain

$$
\operatorname{Res}(-1)=\frac{d z}{12}\left(-\frac{1}{2}+F(r z+i \pi, 0)\right) .
$$

Formulae $(9 \cdot 5),(9 \cdot 6),(9 \cdot 7),(9 \cdot 3),(9 \cdot 4)$ end the proof of Lemma 9.2.

Now we have to study the terms $F(*, s)$ with $s=0,1,2$. First we have

$$
F(r z+i \pi, 0)=\sum_{m=1}^{\infty}(-1)^{m} \exp (-m r z)=-\frac{1}{1+\exp (r z)}=-\frac{1}{2}+O\left(r n^{-1 / 2}\right),
$$

for $|y| \leqslant y_{1}$. For the contribution of $F(r z+i \pi, 1)$ we have

$$
\sum_{m=1}^{\infty} \frac{(-1)^{m+1}}{2 m} \exp (-m r z)=\frac{1}{2} \log (1+\exp (-r z))=\frac{1}{2} \log 2+O(r|z|) .
$$

The contribution of $F(r z+i \pi, 2)$ is more difficult to handle because of the factor $(d z)^{-1}$. We will prove the following lemma 
Lemma 9.3. For $|y| \leqslant y_{1}$, we have for $d \leqslant n^{1 / 4-2 \varepsilon}$

$$
\frac{1}{d z} F(r z+i \pi, 2)=-\frac{\pi^{2}}{12 d z}+\frac{r}{d} \log 2+O\left(d^{-1} n^{-2 \varepsilon}\right) .
$$

Proof. Let $M$ be an odd integer. We have

$$
\frac{1}{d z} F(r z+i \pi, 2)=\frac{1}{d z} \sum_{m=1}^{M} \frac{(-1)^{m} \exp (-m r z)}{m^{2}}+\sum_{m=M+1}^{+\infty} \frac{(-1)^{m} \exp (-m r z)}{d z m^{2}}=T_{1}+T_{2},
$$

say. We begin with $T_{2}$. Since the sum is absolutely convergent, we can regroup the terms $m=2 \ell$ with the terms $m=2 \ell+1$

$$
\begin{aligned}
\left|T_{2}\right| & \leqslant \frac{1}{d|z|} \sum_{\ell=\frac{M+1}{2}}^{+\infty} \exp \left(-2 \ell x_{0}\right)\left|\frac{1}{4 \ell^{2}}-\frac{\exp (-r z)}{(2 \ell+1)^{2}}\right| \leqslant \frac{1}{d|z|} \sum_{\ell=\frac{M+1}{2}}^{+\infty}\left|\frac{1}{4 \ell^{2}}-\frac{1+O(r|z|)}{(2 \ell+1)^{2}}\right| \\
& \leqslant \frac{1}{d|z|} \sum_{\ell=\frac{M+1}{2}}^{+\infty}\left(\frac{4 \ell+1}{4 \ell^{2}(2 \ell+1)^{2}}+O\left(\frac{r|z|}{(2 \ell+1)^{2}}\right)\right) \ll \frac{1}{d|z| M^{2}}+\frac{r}{d M} .
\end{aligned}
$$

This leads us to choose $M$ the smallest odd integer $\geqslant n^{1 / 4+\varepsilon}$. For $T_{1}$ we use the fact that $\exp (-m r z)$ is near 1 if $M$ is not too large. Let $J \in \mathbb{N}$ to be specified later:

$$
T_{1}=\frac{1}{d z} \sum_{m=1}^{M} \frac{(-1)^{m}}{m^{2}}\left(1-m r z+\sum_{j=2}^{J-1} \frac{(-1)^{j} m^{j} r^{j} z^{j}}{j !}+O\left(m^{J} r^{J}|z|^{J}\right)\right) .
$$

By the same type of argument as for $T_{2}$ (which are standard manipulations on alternating series) we show that

$$
\frac{1}{d z} \sum_{m=1}^{M} \frac{(-1)^{m}}{m^{2}}=\frac{1}{d z} \sum_{m=1}^{+\infty} \frac{(-1)^{m}}{m^{2}}+O\left(\frac{1}{d|z| M^{2}}\right)=-\frac{\pi^{2}}{12 d z}+O\left(\frac{1}{d|z| M^{2}}\right),
$$

and

$$
\frac{r}{d} \sum_{m=1}^{M} \frac{(-1)^{m}}{m}=\frac{r}{d} \sum_{m=1}^{+\infty} \frac{(-1)^{m}}{m}+O\left(\frac{r}{d M}\right)=-\frac{r}{d} \log 2+O\left(\frac{r}{d M}\right) .
$$

Next, for each $2 \leqslant j \leqslant J-1$ we have (recall that $r \leqslant d \leqslant n^{1 / 4-2 \varepsilon}$ )

$$
\frac{r^{j}|z|^{j-1}}{d}\left|\sum_{m=1}^{M}(-1)^{m} m^{j-2}\right| \ll \frac{r^{j}|z|^{j-1} M^{j-2}}{d} \ll \frac{n^{-(2+j) \varepsilon}}{d} .
$$

Finally for the last error term we have:

$$
r^{J}|z|^{J-1} d^{-1} \sum_{m=1}^{M} m^{J-2} \ll(M|z|)^{J-1} r^{J} d^{-1} \ll n^{\frac{1}{4}-J \varepsilon} d^{-1},
$$

which is small enough for $J=\left\lceil\frac{5}{\varepsilon}\right\rceil$.

This ends the proof of Lemma 9.3.

If we insert (9.8) and Lemma 9.3 in Lemma 9.2 and don't forget (9.1) we obtain Lemma 9.1 in the case $r \neq d$. 


\section{The term $S_{0}(0)$, end of the proof of Theorem 1.1}

Recall that

$$
h(\exp (-z))=\exp \left(\frac{\pi^{2}}{12 z}-\frac{1}{2} \log 2+O(|z|)\right),
$$

if $z \rightarrow 0$ with $|\arg z| \leqslant \kappa<\pi / 2$. By Lemma 9.1 we have for $|y| \leqslant y_{1}$ :

$$
\begin{aligned}
h(\exp (-z)) \prod_{r \in \mathcal{D}} U_{r}(z) & =\exp \left(\frac{\pi^{2}}{12 z}\left(1+\frac{|\mathcal{D}|}{d}\right)+\frac{|\mathcal{D}|}{2} \log z-\frac{\log 2}{2}\right. \\
& \left.+\sum_{r \in \mathcal{D}}\left(\frac{r}{d}-1\right) \log 2+\frac{|\mathcal{D}|}{2} \log \left(\frac{d}{\pi}\right)+O\left(n^{-\varepsilon}\right)\right) .
\end{aligned}
$$

As in the case $\mathcal{D}=\{1, \ldots, d\}$ we insert above the two formulae:

$$
\frac{1}{z}=\frac{1}{x_{0}}-\frac{i y}{x_{0}^{2}}-\frac{y^{2}}{x_{0}^{3}}+O\left(\frac{|y|^{3}}{x_{0}^{4}}\right) \text { and } \log z=\log x_{0}+O\left(\frac{|y|}{x_{0}}\right)
$$

and we apply Lemma 4.1:

$$
S_{0}(0)=\frac{1}{2 \pi} \int_{-y_{1}}^{y_{1}} \exp \left(C_{\mathcal{D}}+i y \tilde{B}_{\mathcal{D}}-y^{2} A_{\mathcal{D}}\right) \mathrm{d} y,
$$

with

$$
\begin{gathered}
\tilde{B}_{\mathcal{D}}=-\frac{\pi^{2}}{12 x_{0}^{2}}\left(1+\frac{|\mathcal{D}|}{d}\right)+\frac{C_{2}|\mathcal{D}|}{d x_{0}^{2}}+\frac{|\mathcal{D}| k_{0} \log 2}{x_{0}}-d k_{0} \sum_{r \in \mathcal{D}}\left(N_{r}-k_{0}\right)+n-R_{\mathcal{D}}-Q_{\mathcal{D}} \\
A_{\mathcal{D}}=\frac{\pi^{2}}{12 x_{0}^{3}}\left(1+\frac{|\mathcal{D}|}{d}\right)-\frac{C_{2}|\mathcal{D}|}{d x_{0}^{3}}-\frac{|\mathcal{D}| k_{0} \log 2}{x_{0}^{2}}-\frac{d|\mathcal{D}| k_{0}^{2}}{2 x_{0}}
\end{gathered}
$$

Since $k_{0} d x_{0}=\log 2$, and $C_{2}=\frac{\pi^{2}}{12}-\frac{(\log 2)^{2}}{2}, A_{\mathcal{D}}, \tilde{B}_{\mathcal{D}}$ and $C_{\mathcal{D}}$ may be simplified :

$$
A_{\mathcal{D}}=\frac{\pi^{2}}{12 x_{0}^{3}}-\frac{|\mathcal{D}|(\log 2)^{2}}{d x_{0}^{3}}=\frac{2 \sqrt{3} n^{\frac{3}{2}}}{\pi}\left(1-\frac{12|\mathcal{D}|(\log 2)^{2}}{d \pi^{2}}\right) .
$$

Recall that $k_{0}=\frac{2 \sqrt{3 n} \log 2}{\pi d}$ and $\left|N_{r}-k_{0}\right| \leqslant \frac{n^{\frac{1}{4}} \sqrt{\log n}}{d^{1 / 3}|\mathcal{D}|^{2 / 3} w(n)}$.

By Lemma 2.2 of [6], for $|y| \leqslant y_{1}$, we have

$$
y \tilde{B}_{\mathcal{D}}=-2 y d k_{0} \sum_{r \in \mathcal{D}}\left(N_{r}-k_{0}\right)+O\left(n^{-\varepsilon / 3}\right)=y B_{\mathcal{D}}+O\left(n^{-\varepsilon / 3}\right),
$$

say. 
We end the computations as in the case $\mathcal{D}=\{1, \ldots, d\}$. Finally we obtain

$$
\Pi_{d}^{*}\left(n, \mathcal{R}_{\mathcal{D}}\right)=(1+o(1)) \frac{\delta}{2 \pi} \sqrt{\frac{\pi}{A_{\mathcal{D}}}} \exp \left(C_{\mathcal{D}}-\frac{B_{\mathcal{D}}^{2}}{4 A_{\mathcal{D}}}\right) .
$$

To simplify $C_{\mathcal{D}}$ we need some more precise estimations of $R_{\mathcal{D}}$ and $Q_{\mathcal{D}}$ :

$$
\begin{gathered}
x_{0} R_{\mathcal{D}}=x_{0} k_{0} \sum_{r \in \mathcal{D}} r+O\left(d^{2 / 3}|\mathcal{D}|^{1 / 3} n^{-1 / 4+\varepsilon}\right), \\
Q_{\mathcal{D}} x_{0}=\frac{d x_{0}}{2} \sum_{r \in \mathcal{D}}\left(N_{r}-k_{0}\right)^{2}+k_{0} d x_{0} \sum_{r \in \mathcal{D}}\left(N_{r}-k_{0}\right)+\frac{x_{0} d k_{0}^{2}|\mathcal{D}|}{2}-\frac{d|\mathcal{D}| k_{0} x_{0}}{2}+O\left(d n^{-1 / 4+\varepsilon}\right) .
\end{gathered}
$$

It remains to insert these different formulae in (10.4) to finish the proof of Theorem 1.1.

\section{Local stability of $\Pi_{d}^{*}\left(n, \mathcal{R}_{\mathcal{D}}\right)$}

In this section we settle a result analogous to Corollary 9.1 of [5]. If $\mathcal{R}_{\mathcal{D}}=\left\{N_{r}: r \in \mathcal{D}\right\}$ and $\mathcal{R}_{\mathcal{D}}^{*}=\left\{N_{r}^{*}: r \in \mathcal{D}\right\}$ are two sets of integers satisfying (1.1) and such that $N_{r}^{*}$ is near $N_{r}$ on average then in the estimation of $\Pi_{d}^{*}\left(n, \mathcal{R}_{D}\right)$ given by Theorem 1.1, we may replace the $N_{r}$ by $N_{r}^{*}$. Like in [5] this corollary will be useful for the proofs of the different corollaries announced in the introduction of [6].

Corollary 11.1. Let $0<\varepsilon<10^{-2}, n \geqslant n_{0}, d^{3}|\mathcal{D}| \leqslant n^{1 / 2-3 \varepsilon}$ and two sets $\mathcal{R}_{\mathcal{D}}=\left\{N_{r}\right.$ : $r \in \mathcal{D}\} \in \mathbb{Z}^{|\mathcal{D}|}, \mathcal{R}_{\mathcal{D}}^{*}=\left\{N_{r}^{*}: r \in \mathcal{D}\right\} \in \mathbb{R}^{|\mathcal{D}|}$ such that

(i) (1.1) is satisfied for $\mathcal{R}_{\mathcal{D}}$;

(ii) $\left|N_{r}-k_{0}\right| \leqslant \frac{n^{1 / 4} \sqrt{\log n}}{d^{1 / 3}|\mathcal{D}|^{2 / 3} w(n)}$ for all $r \in \mathcal{D}$ where $w(n)$ is a non decreasing function such that $\lim _{u \rightarrow \infty} w(u)=\infty$;

(iii) $\sum_{r \in \mathcal{D}}\left|N_{r}-N_{r}^{*}\right| \leqslant \delta+|\mathcal{D}|-1, \quad \sum_{r \in \mathcal{D}}\left|N_{r}-N_{r}^{*}\right|^{2} \leqslant \delta^{2}+|\mathcal{D}|-1$.

Then we have

$$
\begin{aligned}
\Pi_{d}^{*}\left(n, \mathcal{R}_{D}\right) & =q(n) \frac{\delta(1+o(1))}{\sqrt{1-\frac{12|\mathcal{D}|(\log 2)^{2}}{d \pi^{2}}}}\left(\frac{d}{2 \sqrt{3 n}}\right)^{|\mathcal{D}| / 2} \\
& \times \exp \left(-\frac{2 \sqrt{3}(\log 2)^{2}}{\pi\left(1-\frac{12|\mathcal{D}|(\log 2)^{2}}{d \pi^{2}}\right) \sqrt{n}}\left(\sum_{r \in \mathcal{D}}\left(N_{r}^{*}-k_{0}\right)\right)^{2}-\frac{\pi d}{2 \sqrt{3 n}} \sum_{r \in \mathcal{D}}\left(N_{r}^{*}-k_{0}\right)^{2}\right) .
\end{aligned}
$$

Proof. By (iii), $\sum_{r \in \mathcal{D}}\left|N_{r}-N_{r}^{*}\right| \leqslant 2 d$ and we have

$$
\begin{aligned}
\left|\left(\sum_{r \in \mathcal{D}}\left(N_{r}-k_{0}\right)\right)^{2}-\left(\sum_{r \in \mathcal{D}}\left(N_{r}^{*}-k_{0}\right)\right)^{2}\right| & \leqslant\left(\sum_{r \in \mathcal{D}}\left|N_{r}-N_{r}^{*}\right|\right)\left(2 \sum_{r \in \mathcal{D}}\left|N_{r}-k_{0}\right|+\sum_{r \in \mathcal{D}}\left|N_{r}-N_{r}^{*}\right|\right) \\
& =O\left(d^{2 / 3}|\mathcal{D}|^{1 / 3} \frac{n^{1 / 4} \sqrt{\log n}}{w(n)}+d^{2}\right)=o\left(n^{1 / 2}\right) .
\end{aligned}
$$

Similarly we have using also $\delta \leqslant|\mathcal{D}|+1\left(\right.$ since $\delta \leqslant \min _{a \in \mathcal{D}^{c}} a$ if $\left.\mathcal{D}^{c} \neq \varnothing\right)$

$$
\begin{aligned}
\left|\sum_{r \in \mathcal{D}}\left(N_{r}-k_{0}\right)^{2}-\sum_{r \in \mathcal{D}}\left(N_{r}^{*}-k_{0}\right)^{2}\right| & \leqslant \sum_{r \in \mathcal{D}}\left|N_{r}-N_{r}^{*}\right|\left(2\left|N_{r}-k_{0}\right|+\left|N_{r}-N_{r}^{*}\right|\right) \\
& \ll(\delta+|\mathcal{D}|-1)|\mathcal{D}|^{-2 / 3} d^{-1 / 3} \frac{n^{1 / 4} \sqrt{\log n}}{w(n)}+\delta^{2}+|\mathcal{D}| \\
& \ll|\mathcal{D}|^{1 / 3} d^{-1 / 3} \frac{n^{1 / 4} \sqrt{\log n}}{w(n)}+\delta^{2}+|\mathcal{D}|=o\left(\frac{\sqrt{n}}{d}\right) .
\end{aligned}
$$

This ends the proof of Corollary 11.1. 


\section{On the normal order of the numbers of parts: proof of Corollary 1.2 of [6]}

Let $C_{\mathcal{D}}=\left\lceil\frac{2 \sqrt{3} \log 2}{\pi} \frac{\sqrt{n}}{d^{2}}-\frac{n^{1 / 4} \sqrt{\log n}}{d^{4 / 3}|\mathcal{D}|^{2 / 3} w(n)}\right\rceil d$ and $D_{\mathcal{D}}=\left\lfloor\frac{2 \sqrt{3} \log 2}{\pi} \frac{\sqrt{n}}{d^{2}}+\frac{n^{1 / 4} \sqrt{\log n}}{d^{4 / 3}|\mathcal{D}|^{2 / 3} w(n)}\right\rfloor d$.

To prove Corollary 1.2 of [6], it is sufficient to show that

$$
S^{*}:=\sum_{\substack{N_{r} \in\left[C_{\mathcal{D}}, D_{\mathcal{D}}\left[ \\r \in \mathcal{D} \\ \mathcal{R}_{\mathcal{D}} \equiv n(\bmod \delta)\right.\right.}} \Pi_{d}^{*}\left(n, \mathcal{R}_{\mathcal{D}}\right)=q(n)(1+o(1)) .
$$

As in [5] p. 82, we have to remove the dependence between $n$ and the $N_{r}$ given by the congruence condition modulo $\delta$. If $1 \notin \mathcal{D}$ then $\delta=1$, there are no difficulty and we take $N_{r}^{*}=N_{r}$ for all $r \in \mathcal{D}$. If $1 \in \mathcal{D}$ we write $N_{1}^{*}=\left\lfloor\frac{N_{1}}{\delta}\right\rfloor \delta$ and $N_{r}^{*}=N_{r}$ for $r \in \mathcal{D} \backslash\{1\}$. Now we will suppose that $1 \in \mathcal{D}$, the proof of the other case being similar. Next we apply Corollary 11.1 with these two sets:

$$
\begin{aligned}
S^{*} & =(1+o(1)) q(n) \frac{\delta}{\sqrt{1-\frac{12|\mathcal{D}|(\log 2)^{2}}{d \pi^{2}}}}\left(\frac{d}{2 \sqrt{3 n}}\right)^{|\mathcal{D}| / 2} \\
& \times \sum_{\substack{C_{\mathcal{D}} \leqslant N_{1} \delta<D_{\mathcal{D}} \\
N_{r} \in\left[C_{\mathcal{D}}, D_{\mathcal{D}}[ \\
r \in \mathcal{D} \backslash\{1\}\right.}} \exp \left(-\frac{2 \sqrt{3}(\log 2)^{2}}{\pi\left(1-\frac{12|\mathcal{D}|(\log 2)^{2}}{d \pi^{2}}\right) \sqrt{n}}\left(\delta N_{1}-k_{0}+\sum_{r \in \mathcal{D} \backslash\{1\}}\left(N_{r}-k_{0}\right)\right)^{2}\right. \\
& \left.-\frac{\pi d}{2 \sqrt{3 n}}\left(\left(\delta N_{1}-k_{0}\right)^{2}+\sum_{r \in \mathcal{D} \backslash\{1\}}\left(N_{r}-k_{0}\right)^{2}\right)\right) .
\end{aligned}
$$

We apply again Corollary 11.1 with $N_{i}^{*}=t_{i}$ so that $\left|t_{i}-N_{i}\right| \leqslant 1$ for $i \in \mathcal{D}$, we easily see that

$$
\left|\delta t_{1}^{\prime}-\delta N_{1}\right|^{2}+\sum_{r \in \mathcal{D} \backslash\{1\}}\left(t_{r}-N_{r}\right)^{2} \leqslant \delta^{2}+|\mathcal{D}|-1 .
$$

Thus we have (after replacing $\delta t_{1}^{\prime}$ by $t_{1}$ in the integral) :

$$
\begin{aligned}
S^{*} & =(1+o(1)) q(n) \frac{1}{\sqrt{1-\frac{12|\mathcal{D}|(\log 2)^{2}}{d \pi^{2}}}}\left(\frac{d}{2 \sqrt{3 n}}\right)^{|\mathcal{D}| / 2} \\
& \times \int_{C_{\mathcal{D}}}^{D_{\mathcal{D}}} \cdots \int_{C_{\mathcal{D}}}^{D_{\mathcal{D}}} \exp \left(-\frac{2 \sqrt{3}(\log 2)^{2}}{\pi\left(1-\frac{12|\mathcal{D}|(\log 2)^{2}}{d \pi^{2}}\right) \sqrt{n}}\left(\sum_{r \in \mathcal{D}}\left(t_{r}-k_{0}\right)\right)^{2}\right. \\
& \left.-\frac{\pi d}{2 \sqrt{3 n}}\left(\sum_{r \in \mathcal{D}}\left(t_{r}-k_{0}\right)^{2}\right)\right) \prod_{r \in \mathcal{D}} \mathrm{d} t_{r} .
\end{aligned}
$$

Lemma 12.1. We have

$$
\begin{aligned}
S^{*} & =(1+o(1)) q(n) \frac{1}{\sqrt{1-\frac{12|\mathcal{D}|(\log 2)^{2}}{d \pi^{2}}}}\left(\frac{d}{2 \sqrt{3 n}}\right)^{|\mathcal{D}| / 2} \\
& \times \int_{-\infty}^{+\infty} \cdots \int_{-\infty}^{+\infty} \exp \left(-\frac{2 \sqrt{3}(\log 2)^{2}}{\pi\left(1-\frac{12|\mathcal{D}|(\log 2)^{2}}{d \pi^{2}}\right) \sqrt{n}}\left(\sum_{r \in \mathcal{D}}\left(t_{r}-k_{0}\right)\right)^{2}\right. \\
& \left.-\frac{\pi d}{2 \sqrt{3 n}}\left(\sum_{r \in \mathcal{D}}\left(t_{r}-k_{0}\right)^{2}\right)\right) \prod_{r \in \mathcal{D}} \mathrm{d} t_{r} \\
& +O\left(q(n) \frac{w(n)|\mathcal{D}|^{5 / 3}}{d^{1 / 6} \sqrt{\log n}} \exp \left(-\frac{\pi d^{1 / 3} \log n}{2 \sqrt{3}|\mathcal{D}|^{4 / 3} w^{2}(n)}\right)\right) .
\end{aligned}
$$


Proof. We have to consider the contribution of terms of type $\int_{D_{\mathcal{D}}}^{\infty} \int_{C_{\mathcal{D}}}^{D_{\mathcal{D}}} \cdots \int_{C_{\mathcal{D}}}^{D_{\mathcal{D}}} \cdots$. Clearly it is less than

$$
\int_{D_{\mathcal{D}}}^{\infty} \int_{C_{\mathcal{D}}}^{D_{\mathcal{D}}} \cdots \int_{C_{\mathcal{D}}}^{D_{\mathcal{D}}} \exp \left(-\frac{\pi d}{2 \sqrt{3 n}}\left(\sum_{r \in \mathcal{D}}\left(t_{r}-k_{0}\right)^{2}\right)\right) \prod_{r \in \mathcal{D}} \mathrm{d} t_{r}
$$

But

$$
\int_{C_{\mathcal{D}}}^{D_{\mathcal{D}}} \exp \left(-\frac{\pi d}{2 \sqrt{3 n}}\left(t_{r}-k_{0}\right)^{2}\right) \mathrm{d} t_{r} \leqslant \int_{-\infty}^{+\infty} \exp \left(-\frac{\pi d t_{r}^{2}}{2 \sqrt{3 n}}\right) \mathrm{d} t_{r}=\sqrt{\frac{2 \sqrt{3 n}}{d}}
$$

and

$$
\begin{aligned}
\int_{D_{\mathcal{D}}}^{\infty} \exp \left(-\frac{\pi d}{2 \sqrt{3 n}}\left(t_{r}-k_{0}\right)^{2}\right) \mathrm{d} t_{r} & \leqslant \int_{D_{\mathcal{D}}}^{\infty} \exp \left(-\frac{\pi d}{2 \sqrt{3 n}}\left(D_{\mathcal{D}}-k_{0}\right)\left(t_{r}-k_{0}\right)\right) \mathrm{d} t_{r} \\
& =\frac{2 \sqrt{3 n}}{\pi d\left(D_{\mathcal{D}}-k_{0}\right)} \exp \left(-\frac{\pi d}{2 \sqrt{3 n}}\left(D_{\mathcal{D}}-k_{0}\right)^{2}\right) .
\end{aligned}
$$

We have $d^{4 / 3}|\mathcal{D}|^{2 / 3}=d^{8 / 6}|\mathcal{D}|^{1 / 2}|\mathcal{D}|^{1 / 6} \leqslant d^{9 / 6}|\mathcal{D}|^{1 / 2}=\left(d^{3}|\mathcal{D}|\right)^{1 / 2} \leqslant n^{1 / 4-\varepsilon}$. Thus the contribution of some term of type $\int_{D_{\mathcal{D}}}^{\infty} \int_{C_{\mathcal{D}}}^{D_{\mathcal{D}}} \cdots \int_{C_{\mathcal{D}}}^{D_{\mathcal{D}}} \cdots$ to $S^{*}$ is

$$
O\left(q(n) \frac{w(n)|\mathcal{D}|^{2 / 3}}{d^{1 / 6} \sqrt{\log n}} \exp \left(-\frac{\pi d^{1 / 3} \log n}{2 \sqrt{3}|\mathcal{D}|^{4 / 3} w^{2}(n)}\right)\right) .
$$

Since there are $2|\mathcal{D}|$ such error terms we obtain Lemma 12.1. It remains to compute the main term to finish the proof of Corollary 1.2 of [6]. We remark that

$\exp \left(-\frac{2 \sqrt{3}(\log 2)^{2}}{\pi\left(1-\frac{12|\mathcal{D}|(\log 2)^{2}}{d \pi^{2}}\right) \sqrt{n}}\left(\sum_{r \in \mathcal{D}}\left(t_{r}-k_{0}\right)\right)^{2}-\frac{\pi d}{2 \sqrt{3 n}}\left(\sum_{r \in \mathcal{D}}\left(t_{r}-k_{0}\right)^{2}\right)\right)=\exp \left(-\frac{1}{2} T^{t} M T\right)$,

with $T=\left(\begin{array}{c}t_{1}-k_{0} \\ t_{2}-k_{0} \\ \vdots \\ t_{\mathcal{D}}-k_{0}\end{array}\right) \in \mathbb{R}^{|\mathcal{D}|}$ and $M$ is the symmetric matrix $M=\left(m_{i j}\right)_{1 \leqslant i, j \leqslant|\mathcal{D}|}$ defined by

$$
\begin{aligned}
& \frac{m_{i i}}{2}=\frac{2 \sqrt{3}(\log 2)^{2}}{\pi\left(1-\frac{12|\mathcal{D}|(\log 2)^{2}}{d \pi^{2}}\right) \sqrt{n}}+\frac{\pi d}{2 \sqrt{3 n}}=: V+U \quad(1 \leqslant i \leqslant|\mathcal{D}|) \\
& \frac{m_{i j}}{2}=\frac{m_{j i}}{2}=\frac{2 \sqrt{3}(\log 2)^{2}}{\pi\left(1-\frac{12|\mathcal{D}|(\log 2)^{2}}{d \pi^{2}}\right) \sqrt{n}}=: V \quad(1 \leqslant i<j \leqslant|\mathcal{D}|) .
\end{aligned}
$$

A classical result on determinant announces that

$$
\operatorname{det} M=2^{|\mathcal{D}|} U^{|\mathcal{D}|-1}(U+|\mathcal{D}| V)=\left(\frac{\pi d}{\sqrt{3 n}}\right)^{|\mathcal{D}|}\left(1-\frac{12|\mathcal{D}|(\log 2)^{2}}{d \pi^{2}}\right)^{-1} .
$$

Thus the function (12.2) is proportional to the density of the law of a Gaussian vector with covariance matrix $M^{-1}$. We deduce that

$$
\begin{aligned}
& \int_{-\infty}^{\infty} \cdots \int_{-\infty}^{\infty} \exp \left(-\frac{2 \sqrt{3}(\log 2)^{2}}{\pi\left(1-\frac{12|\mathcal{D}|(\log 2)^{2}}{d \pi^{2}}\right) \sqrt{n}}\left(\sum_{r \in \mathcal{D}}\left(t_{r}-k_{0}\right)\right)^{2}-\frac{\pi d}{2 \sqrt{3 n}}\left(\sum_{r \in \mathcal{D}}\left(t_{r}-k_{0}\right)^{2}\right)\right) \prod_{r \in \mathcal{D}} d_{t_{r}} \\
& =(2 \pi)^{|\mathcal{D}| / 2 \sqrt{\operatorname{det} M^{-1}}} .
\end{aligned}
$$

This ends the proof of Corollary 1.2 of [6]. 


\section{Unequal partitions with equilibrated residue classes: proof of Corollary 1.3 of $[6]$}

For $1 \leqslant a<b \leqslant d$, let $E^{*}(a, b)$ denote the number of unequal partitions of $n$ such that $N_{a}=N_{b}$ :

$$
E^{*}(a, b)=\sum_{\substack{N_{a}=N_{b} \\
n \equiv a N_{a}+b N_{b}(\bmod \delta)}} \Pi_{d}^{*}\left(n, \mathcal{R}_{\{a, b\}}\right)=\sum_{\begin{array}{c}
N_{a}=N_{b} \\
N_{a} \in[C, D] \\
n \equiv a N_{a}+b N_{b}(\bmod \delta)
\end{array}} \Pi_{d}^{*}\left(n, \mathcal{R}_{\{a, b\}}\right)+o(q(n)),
$$

by Corollary 1.2 of [6] applied with $w(n)=2^{-2 / 3} \log \log n$,

$$
C=\left\lceil\frac{2 \sqrt{3} \log 2}{\pi} \frac{\sqrt{n}}{d^{2}}-\frac{n^{1 / 4} \sqrt{\log n}}{d^{4 / 3} \log \log n}\right\rceil d \text { and } D=\left\lfloor\frac{2 \sqrt{3} \log 2}{\pi} \frac{\sqrt{n}}{d^{2}}+\frac{n^{1 / 4} \sqrt{\log n}}{d^{4 / 3} \log \log n}\right\rfloor d .
$$

Next we apply Theorem 1.1 and Corollary 11.1. Here again we have to remove the condition $n \equiv a N_{a}+b N_{b}(\bmod \delta)$ otherwise $N_{a}, N_{b}, n$ wouldn't be independent. If $d \geqslant 5$ then $\delta=1$. For $2 \leqslant d \leqslant 4$, the problematic cases are $(a, b, d) \in\{(1,2,2),(1,2,3),(1,3,4)\}$. We will handle these cases later. Suppose now that $\delta=1$.

$$
\begin{aligned}
E^{*}(a, b) & =q(n) \frac{1}{\sqrt{1-\frac{24(\log 2)^{2}}{d \pi^{2}}}}\left(\frac{d}{2 \sqrt{3 n}}\right) \\
& \times \int_{C}^{D} \exp \left[-\left(\frac{8 \sqrt{3}(\log 2)^{2}}{\pi\left(1-\frac{24(\log 2)^{2}}{d \pi^{2}}\right) \sqrt{n}}+\frac{\pi d}{\sqrt{3 n}}\right)\left(t-k_{0}\right)^{2}\right] \mathrm{d} t+o(q(n)) \\
& =\sqrt{\frac{d}{4 \sqrt{3 n}}} q(n)+o(q(n)) .
\end{aligned}
$$

As in the proof of Corollary 1.2 of [6] we show that the contributions of $\int_{D}^{\infty} \cdots$ and $\int_{-\infty}^{C}$ are small enough. When $\delta>1$, as explained in [5] we fixe some congruence conditions on $N_{a}$ and $N_{b}$ and next do quite the same computations.

\section{Comparison between the number of parts in two residue classes: proof of Corollary 1.4 of [6]}

We proceed in the same way as in the previous section. Le $\Delta \in\{0,1\}$. We have to estimate

$$
T^{*}(a, b)=\sum_{\substack{N_{a} \geqslant N_{b}+\Delta \\ n \equiv R_{\{a, b\}}(\bmod \delta)}} \Pi_{d}^{*}\left(n, \mathcal{R}_{\{a, b\}}\right) .
$$

We take $\Delta=0$ to examine the unequal partitions with $N_{a} \geqslant N_{b}$ and $\Delta=1$ for the unequal partitions with $N_{a}>N_{b}$. In the same way as in the proof of Corollary 1.3 of [6], we obtain

$$
\begin{aligned}
T^{*}(a, b) & =q(n) \frac{1}{\sqrt{1-\frac{24(\log 2)^{2}}{d \pi^{2}}}}\left(\frac{d}{2 \sqrt{3 n}}\right) \\
& \times \int_{-\infty}^{\infty} \int_{t_{b}}^{\infty} \exp \left[-\frac{2 \sqrt{3}(\log 2)^{2}}{\pi\left(1-\frac{24(\log 2)^{2}}{d \pi^{2}}\right) \sqrt{n}}\left(t_{a}+t_{b}-2 k_{0}\right)^{2}\right. \\
& -\frac{\pi d}{2 \sqrt{3 n}}\left(\left(t_{a}-k_{0}\right)^{2}+\left(t_{b}-k_{0}\right)^{2}\right] \mathrm{d} t_{a} \mathrm{~d} t_{b}+o(q(n)) .
\end{aligned}
$$

In the same way we find a similar formula for $T^{*}(b, a)$. This proves that $T^{*}(a, b)=$ $q(n) / 2+o(q(n))$. 


\section{On the $d$ regularity of the unequal partitions: proof of Corollary 1.5 of [6]}

Now we suppose that $d$ is fixed in order to use Corollary 1.3 of [6] uniformly. We now study for $\Delta=0$ or 1 :

$$
W^{*}(a):=\sum_{\substack{N_{1}, \ldots, N_{d} \\ n \equiv R(\bmod d) \\ N a \geqslant \Delta+\max _{b \neq a} N_{b}}} \Pi_{d}^{*}(n, \mathcal{R})
$$

We proceed as [5] Sections 12, 13 and like the previous paragraphs of this present paper :

$$
W^{*}(a)=o(q(n))+\frac{d}{\sqrt{1-\frac{12(\log 2)^{2}}{\pi^{2}}}}\left(\frac{d}{2 \sqrt{3 n}}\right)^{d / 2} \int \cdots \int_{t_{a} \geqslant \max _{b \neq a} t_{b}} f\left(t_{1}, \ldots, t_{d}\right) \mathrm{d} t_{1} \cdots \mathrm{d} t_{d}
$$

with

$$
f\left(t_{1}, \ldots, t_{d}\right)=\exp \left\{-\frac{2 \sqrt{3} \log ^{2} 2}{\pi\left(1-\frac{12(\log 2)^{2}}{\pi^{2}}\right) \sqrt{n}}\left(\sum_{r=1}^{d}\left(t_{r}-k_{0}\right)\right)^{2}-\frac{\pi d}{2 \sqrt{3 n}} \sum_{r=1}^{d}\left(t_{r}-k_{0}\right)^{2}\right\} .
$$

By Corollary 1.3 of [6] applied $d$ times (it is why $d$ is fixed), we have

$$
\sum_{a=1}^{d} W^{*}(a)=q(n)+o(q(n))
$$

Since $f\left(t_{1}, \ldots, t_{d}\right)$ is symmetrical the above terms are asymptotically equal :

$$
W^{*}(a)=\left(\frac{1}{d}+o(1)\right) q(n),
$$

as it was conjectured in [4] p.334 and in the introduction of [3].

The proof of the second assertion of Corollary 1.5 of [6] is similar. We have only to replace the condition $N_{a} \geqslant \max _{b \neq a} N_{b}$ by $N_{\sigma(1)} \geqslant N_{\sigma(2)} \geqslant \cdots \geqslant N_{\sigma(d)}$.

\section{References}

[1] G. E. Andrews, The Theory of Partitions, Cambridge University Press (1984).

[2] T. M. Apostol, Introduction to Analytic Number Theory, Undergraduate Texts in Mathematics, Springer Verlag, (1986).

[3] C. Dartyge and A. Sárközy, Arithmetic properties of summands of partitions, Ramanujan Journal 8 (2004), 199-215.

[4] C. Dartyge, A. Sárközy and M. Szalay, On the distribution of the summands of unequal partitions in residue classes, Acta Math. Hungar. 110 (4) (2006), 323-335.

[5] C. Dartyge and M. Szalay, Dominant residue classes concerning the summands of partitions, Functiones et Approximatio XXXVII.1 (2007), 65-96.

[6] C. Dartyge and M. Szalay, Local distribution of the parts of unequal partitions in arithmetic progressions I, to appear

[7] G. Meinardus, Asymptotische Aussagen über Partitionen, Math. Zeitschr. Bd. 59 (1954), 388398.

[8] G. Tenenbaum, Analyse asymptotique et applications, (Notes de cours) Master de Mathématiques (M2) de l'Université Henri Poincaré-Nancy 1 (2006/2007)

[9] G. Tenenbaum, Introduction à la théorie analytique et probabiliste des nombres, $3^{\mathrm{ème}}$ édition, Collection Échelles, Édition Belin (2008). 
Cécile Dartyge

Institut Élie Cartan

Université Henri Poincaré-Nancy 1,BP 239

54506 Vandouvre Cedex, France

dartyge@iecn.u-nancy.fr
Mihály Szalay

Department of Algebra and Number Theory

Eötvös Loránd University

1117 Budapest, Pázmány Péter sétány 1/C

Hungary

mszalay@cs.elte.hu 